\title{
Simulation and Calibration of Options Prices under a Levy-Type Stochastic Dynamic and Semi Markov Market Switching Regimes Processes
}

\author{
Patrick Assonken ${ }^{1}$, Gangaram Ladde ${ }^{1}$ \\ ${ }^{1}$ Department of Mathematics and Statistics, College of Arts and Sciences, University of South Florida, Tampa, USA \\ Correspondence: Patrick Assonken, Department of Mathematics and Statistics, University of South Florida, Tampa, \\ USA.
}

Received: September 12, 2016

Accepted: October 17, 2016

Available online: October 25, 2016

doi:10.11114/aef.v4i1.1870

URL: http://dx.doi.org/10.11114/aef.v4i1.1870

\begin{abstract}
This work mainly highlights the benefits of derivative pricing in a semi Markov switching market. We explore the main differences between Markov and Semi Markov regime switching models. The three main problems we deal with are, (1) historical parameter calibration through the recently developed LLGMM method, (2) effects of semi Markov parameters on option prices and (3) comparison of Heston model, semi Markov regime model and Markov regime model calibration performances over both sequential option price calibration and the overall implied volatility surface of the market. Employing the LLGMM method, parameters of the spot price process described by a linear Levy-type stochastic differential equation under semi-Markov structural perturbations are calibrated to observed prices. From Fourier space time stepping and Carr and Madan methods, risk neutral parameters of the spot price are calibrated and interpreted. American and European style vanilla and exotic option prices are simulated. The presented results are shown in the context of a piecewise constant semi-Markov intensity matrix approximating a Weibull intensity matrix. We obtain that under the conditional minimum entropy martingale measure, option prices predictably increase as the regime risk increases through the intensity matrix of the semi Markov process. Calibration and simulation results demonstrate noticeable effects of semi-Markov parameters on option prices and a demonstrably better calibration fit of the Black Scholes model over the entire volatility surface, in a market with semi Markov regimes.
\end{abstract}

Keywords: Semi Markov, Regime switching, Option pricing, LLGMM method, Levy processes, Minimum entropy, equivalent martingale measure

\section{Introduction}

Stochastic hybrid models have been used in financial modeling by quite a few authors among which, Assonken \& G.S. (2015); Chourdakis (2005); Hainaut \& Colwell (2014); Kloeden \& Platen (1992); Ling \& G.S. (2010); Siu \& Ladde (2011). This is in response to the well documented limitations of the seminal stock price model by Black $\&$ Scholes (1973). The non-normality of log returns is exhibited by a pronounced skewness and fat tails along with non-constant implied volatility, therefore contradicting modeling assumptions made by Black \& Scholes (1973). Moreover, smiles, smirks and skew empirically observed in the option market are unexplained by the Black Scholes model. Heavy tailed and asymmetric distributions have been successfully applied as a remedy to the log return distribution misfits. However, the skew, smile and smirk are reproduced by exponential Levy models for asset prices with relative success for short to medium maturity (Tankov (2003)). A consensual agreement is that volatility is not constant as assumed by Black \& Scholes (1973). Furthermore, there is strong empirical evidence supporting stochastic volatility. Stochastic volatility and local volatility models have provided a better explanation for many stylized facts of the derivative market and log return times series. However, stochastic regime switching models with random volatility switching from one state to another provide an economically interpretable alternative to stochastic volatility and local volatility models. Regime switching models have been first used in Hamilton (1989) in the context of time series in a two-state market regime. Since then, a slew of regime switching stock price models have ensued (Assonken \& G.S. (2015); Bulla (2006); Chourdakis (2002); Hainaut (2010); Jackson et al. (2007); Naik (1993)). However, most of the models developed are assumed to have Markov states. The convenience of Markov market states stems from the constant conditional intensity matrix of Markov processes which proves to be unrealistic for a market often undergoing 
structural changes. Indeed, under the assumption of constant conditional intensities matrix, the market has the same propensity of switching regime at any given time, regardless of occurring changes. We note that application of Markov regime switching models to financial derivatives is still a work in progress, namely, Markov regime switching exponential Levy models for asset prices with applications in credit risk are used in Hainaut \& Colwell (2014) and an option pricing method under Markov regime switching exponential jump diffusion (Costabile et al. (2014)). On the other hand, semi Markov regime switching models are a relatively unexplored topic (Assonken \& G.S. (2015); Ghosh \& Goswami (2009); Hunt \& Devolder (2011b)). Moreover, simulation methods for option prices from Ghosh \& Goswami (2009); Hunt \& Devolder (2011b) along with continuous and discrete time MCMC calibration method formulated by Hunt \& Devolder (2011b) for semi Markov Black Scholes models of asset prices are developed. Both calibration methods rely on normal likelihood simulation and aren't extensible to other switching exponential Levy models. This is because they either do not have a closed form density function or their known density does not have easy-to-simulate-from conjugate priors. This issue is solved by Assonken \& G.S. (2015), where a closed form expression for the characteristic function of log asset prices is developed. This paved the way for calibration and simulation of option prices induced by an arbitrary exponential Levy price process with closed form characteristic function.

In this paper, we explore four problems of interest: estimation of historical parameters of a semi Markov switching asset price model via LLGMM approach first developed by Otunuga (2014) and Otunuga et al (2017), estimation of the effects of the semi Markov sojourn distribution parameters on option prices, application of Carr \& Madan (1999) and the Fourier space time stepping algorithm of Jackson et al. (2007) to semi Markov modulated stock price processes and comparison of Markov modulated and semi Markov modulated stock price models.

The paper is organized as follows: in Section 2, we define the model along with related filtrations. We use the LLGMM method to estimate the historical parameters of the model illustrated by three case studies in Section 3. Section 4 highlights the effects of risk neutral semi Markov parameters on option prices and volatility surfaces via simulations based on the Carr and Madan method. We also show that we can use the Fourier time stepping method of R Jackson (2009) to price American options and exotic options. Both algorithms are shown to blend naturally in the semi Markovian regime model due to the piecewise constant assumption imposed on the conditional intensity matrix. Section 4 ends with calibrations of Heston model, Markov and semi Markov regime switching Black Scholes models to a couple of option data, and we compare the fit of all models through the residual mean square error risk function. Section 5 concludes our work with a summary and a few problems encountered along the way, which haven't yet found a satisfying resolution.

\section{Preliminary Notations and Definitions of the Model}

Let $T>0$ and $T^{\star}>0$ be the maturity date of an option contract and the time horizon of the market, respectively. We assume that the market is subjected to regime/state structural changes. It is assumed that the market structural states are governed by a semi Markov process $(\theta)_{t \in[0, T]} .\left(\theta_{n}, T_{n}\right)$ is the corresponding Markov renewal process, where $T_{n}$ and $\theta_{n}$ $=\theta_{T n}$ are the time and the state of the process at the n-th regime change. We assume that the structural state domain $E$ of $(\theta)_{t \in[0, T]}$ is finite, and $m=n(E)$. We also denote $\tau_{n}=T_{n+1}-T_{n}$ the sojourn time of the semi Markov process. Let $\left(\beta_{n}\right)_{n \geq 0}$ be a sequence of real nonnegative independent random variables modeling the price jumps at each regime change. We assume that the jump in price only depends on the past and current transition states of the semi Markov process, namely, $\beta_{n}=\beta_{\theta_{n-1}, \theta_{n}}$ with density $g\left(\mid \theta_{n-1}, \theta_{n}\right) . n(t)=\max _{n}\left\{n \in I(0, \infty), T_{n} \leq t\right\}$ denotes last regime change prior to or at time t. Let $\psi\left(\theta_{t}^{-}, d z, d s\right)$ and $v\left(\theta_{s}^{-}, d z\right) d s$ be a Poisson random measure and its intensity measure, respectively. We denote $\bar{\psi}=\psi-v$, the compensated measure of $\psi . G$ and $H$ are smooth functions defined from $\mathbb{R} \times$ $E$ into $\mathbb{R}$ satisfying 


$$
\int_{z \in \mathbb{1}}\left[\left(1+H^{2}(z, j)\right) 1_{||>>1}+1_{|z| \leq 1} G^{2}(z, j)\right] v(j, d z)<\infty, \forall j \in E .
$$

Condition (1) ensures that $\mathrm{H}$ and $\mathrm{G}$ have slow growth enough to allow existence and finiteness of average transformed small and big jumps. It also ensures that the average big jump is finite, which will ensure existence of certain expected values for some versions of $\mathrm{H}$ and $\mathrm{G}$. Such features will be necessary in the remainder of the article as we will apply the isometry property of martingales. Let $L_{t}^{\theta}$ and $\bar{L}_{t}^{\theta}$ be stochastic processes defined by:

$$
\begin{gathered}
L_{t}^{\theta}=\int_{0}^{t} \mu\left(\theta_{s^{-}}, s\right) d s+\int_{0}^{t} \sigma\left(\theta_{s^{-}}, s\right) d B_{s}+\int_{0}^{t} \int_{|k| \leqslant 1} G\left(z, \theta_{s^{-}}\right) \bar{\psi}\left(\theta_{s^{-}}, d z, d s\right) \\
+\int_{0}^{t} \int_{|z|>1} H\left(z, \theta_{s^{-}}\right) \psi\left(\theta_{s^{-}}, d z, d s\right)
\end{gathered}
$$

And

$$
\begin{gathered}
d \tilde{L}_{t}^{\theta}=\int_{0}^{t}\left[\int_{|z| \leq 1}\left(e^{G\left(z, \theta_{s^{-}}\right)}-1-G\left(z, \theta_{s^{-}}\right)\right) v\left(\theta_{s^{-}}, d z\right)+\mu\left(\theta_{s^{-}}, s\right)+\frac{1}{2} \sigma^{2}\left(\theta_{s^{-}}, s\right)\right] d s \\
+\int_{0}^{t} \sigma\left(\theta_{s^{-}}, s\right) d B_{s} \\
+\int_{0}^{t} \int_{|k| \leqslant 1}\left(e^{G\left(z, \theta_{s^{-}}\right)}-1\right) \bar{\psi}\left(\theta_{s^{-}}, d z, d s\right)+\int_{0}^{t} \int_{|z|>1}\left(e^{H\left(z, \theta_{s^{-}}\right)}-1\right) \psi\left(\theta_{s^{-}}, d z, d s\right),
\end{gathered}
$$

respectively. The asset price process $(x(t))_{t \in[0, T]}$ is described by the solution of a following Levy-type stochastic differential equation developed by Assonken \& G.S. (2015); Ladde \& Ladde (2013):

$$
\left\{\begin{array}{l}
d x(t)=x\left(t^{-}\right) d \tilde{L}_{t}^{\theta_{n}}, x\left(T_{n}\right)=x_{n}, t \in\left[T_{n}, T_{n+1}\right), \\
x_{n}=\beta_{n} x\left(T_{n}^{-}, T_{n-1}, x_{n-1}\right), x(0)=x_{0}, \forall n \in I(0, \infty) .
\end{array}\right.
$$

The solution process $x$ defined on each interval $\left[T_{n}, T_{n+1}\right)$ takes the following form found by Assonken \& G.S. (2015) and Ladde \& Ladde (2013):

$$
\left\{\begin{array}{l}
x(t)=x_{n} \exp \left[\int_{T_{n}}^{t} d L_{s}^{\theta_{n}}\right], t \in\left[T_{n}, T_{n+1}\right) \\
x_{n}=\beta_{n} x\left(T_{n}^{-}, T_{n-1}, x_{n-1}\right), \forall n \in I(0, \infty) .
\end{array}\right.
$$

Let $(\Omega, \mathbb{F})$ be the reference measurable space. $\left(\mathbb{H}_{t}\right)_{t \in[0, T]},\left(\mathbb{L}_{t}\right)_{t \in[0, T]}$, and $\left(\mathbb{B}_{n}\right)_{n \geq 0}$, are filtrations generated by the semi Markov process $\theta_{t}$, the Levy processes $L_{s}^{\theta}{ }_{s} \in[0, t], \forall j \in E=\{1,2,3, \ldots, m\}$ and the discrete sequence $\left(\beta_{n}\right)_{n \geq 0}$, , respectively. We also denote $\overline{\mathbb{L}}_{t}=\mathbb{L}_{t} \vee \mathbb{B}_{n(t)}, \overline{\mathbb{G}}_{t}=\overline{\mathbb{L}}_{t} \vee \mathbb{H}_{T}$ and $\mathbb{G}_{t}=\overline{\mathbb{L}}_{t} \vee \mathbb{H}_{t}, \forall t \in\left[0, T^{\star}\right]$. Let $P$ and $Q$ be the historical probability and an equivalent martingale measures as found by Assonken \& G.S. (2015), associated with the price process $\left(x_{t}\right)_{t \in[0, T]}$ defined on the measurable space $(\Omega, \mathbb{F})$, respectively. 


\section{Parameter Estimation Via LLGMM}

We recall the definition of the infinitesimal generator developed by Assonken \& G.S. (2015).

Definition 3.1. Let and $V$ represent the infinitesimal generator of the price process $x(t)$ solution of the SDE (4) and a function such that $V \in \mathcal{C}\left[\square^{+} \times \square^{+} \times \square^{+} \times \square, \square\right]$, with $V$ continuously differentiable in the first and second variables and twice continuously differentiable function in the fourth variable. Let $s \in\left[T_{n}, T_{n+1}\right)$ with $\theta_{T_{n}}=j$. We have,

$$
\begin{gathered}
\mathcal{L} V\left(s, y_{s^{-}}, \theta_{s^{-}}, x_{s^{-}}\right)=\frac{\partial V}{\partial s}+\frac{\partial V}{\partial y}+\left[\mu\left(\theta_{s^{-}}, s\right)+\frac{1}{2} \sigma^{2}\left(\theta_{s^{-}}, s\right)\right] x_{s^{-}} \frac{\partial V}{\partial x}+\frac{1}{2} \sigma^{2}\left(\theta_{s^{-}}, s\right) x_{s^{-}}^{2} \frac{\partial^{2} V}{\partial x^{2}} \\
+\int_{|z| \leq 1}\left[V\left(s, y_{s^{-}}, \theta_{s^{-}}, x_{s^{-}} e^{G\left(z, \theta_{s^{-}}\right)}\right)-V\left(s, y_{s^{-}}, \theta_{s^{-}}, x_{s^{-}}\right)-G\left(z, \theta_{s^{-}}\right) x_{s^{-}} \frac{\partial V}{\partial x}\right] v\left(\theta_{s^{-}}, d z\right) \\
+\int_{|z|>1}\left[V\left(s, y_{s^{-}}, \theta_{s^{-}}, x_{s^{-}} e^{H\left(z, \theta_{s^{-}}\right)}\right)-V\left(s, y_{s^{-}}, \theta_{s^{-}}, x_{s^{-}}\right)\right] v\left(\theta_{s^{-}}, d z\right) \\
+\int_{z \in \square} \sum_{j \neq \theta_{s^{-}}} \lambda_{\theta_{s^{-}}, j}\left(y_{s^{-}}\right)\left[V\left(s, y_{s^{-}}, j, x_{s^{-}} e^{z}\right)-V\left(s, y_{s^{-}}, \theta_{s^{-}}, x_{s^{-}}\right)\right] \bar{b}\left(z \mid \theta_{s^{-}}, j\right) d z .
\end{gathered}
$$

We establish two difference equations that are needed in the LLGMM estimation method.

Lemma 3.1. Let $V \in \mathcal{C}\left[\square^{+} \times \square^{+} \times \square^{+} \times \square, \square\right]$, be continuously differentiable in the first and second variables and twice continuously differentiable function in the fourth variable $P_{M_{n}}^{n}=\left(t_{k}\right)_{k=1}^{M_{n}}: T_{n}=t_{0}<t_{1}<\ldots<t_{M_{n}}=T_{n+1}^{-}$is a partition of the time interval $\left[T_{n}, T_{n+1}\right)$, where $\theta_{n}=j$. The conditional expectation and variance of $V$, associated with a discretized scheme of the transformed stochastic differential equation:

$$
\begin{gathered}
d V\left(s, y_{s}, \theta_{s}, x_{s}\right)=\mathcal{L} V\left(s, y_{s^{-}}, \theta_{s^{-}}, x_{s^{-}}\right)+\sigma\left(\theta_{s^{-}}, s\right) x_{s^{-}} \frac{\partial V}{\partial x} d B_{s} \\
+\int_{|z| \leq 1}\left[V\left(s, y_{s^{-}}, \theta_{s^{-}}, x_{s^{-}} e^{G(z, j)}\right)-V\left(s, y_{s^{-}}, \theta_{s^{-}}, x_{s^{-}}\right)\right] \bar{\psi}(j, d z, d s) \\
+\int_{|z|>1}\left[V\left(s, y_{s^{-}}, \theta_{s^{-}}, x_{s^{-}} e^{H(z, j)}\right)-V\left(s, y_{s^{-}}, \theta_{s^{-}}, x_{s^{-}}\right)\right] \bar{\psi}(j, d z, d s),
\end{gathered}
$$

are:

$$
\begin{aligned}
& E\left[\Delta V\left(t_{k+1}, y_{t_{k+1}}, j, x_{t_{k+1}}\right) \mid \mathbb{G}_{t_{k}}\right]=\mathcal{L} V\left(t_{k}, y_{t_{k}}, j, x_{t_{k}}\right) \Delta t_{k+1} \\
= & E\left[\Delta V\left(t_{k+1}, y_{t_{k+1}}, j, x_{t_{k+1}}\right)-E\left[\Delta V\left(t_{k+1}, y_{t_{k+1}}, j, x_{t_{k+1}}\right) \mid \mathbb{G}_{t_{k}}\right] \mid \mathbb{G}_{t_{k}}\right]^{2}
\end{aligned}
$$

Proof. We apply Euler-Maruyama discretization process as formulated by Kloeden \& Platen (1992), to the transformed Levy-type stochastic differential equation (7) and obtain: 


$$
\begin{gathered}
\Delta V\left(t_{k+1}, y_{t_{k+1}}, j, x_{t_{k+1}}\right)=\mathcal{L} V\left(t_{k}, y_{t_{k}}, j, x_{t_{k}}\right) \Delta t_{k}+\sigma\left(j, t_{k}\right) x_{t_{k}} \frac{\partial V}{\partial x} \Delta B_{t_{k+1}} \\
+\int_{|k| \leq 1}\left[V\left(t_{k}, y_{t_{k}}, j, x_{t_{k}} e^{G(z, j)}\right)-V\left(t_{k}, y_{t_{k}}, j, x_{t_{k}}\right)\right] \bar{\psi}\left(j, d z, \Delta t_{k}\right) \\
+\int_{\mid k>1}\left[V\left(t_{k}, y_{t_{k}}, j, x_{t_{k}} e^{H(z, j)}\right)-V\left(t_{k}, y_{t_{k}}, j, x_{t_{k}}\right)\right] \bar{\psi}\left(j, d z, \Delta t_{k}\right) \text { at } t_{k+1} \in P_{M^{n}}^{n} .
\end{gathered}
$$

Now we apply the conditional mean to the numerical scheme (10) and have:

$$
\begin{gathered}
E\left[\Delta V\left(t_{k+1}, y_{t_{k+1}}, j, x_{t_{k+1}}\right) \mid \mathbb{G}_{t_{k}}\right]=\mathcal{L} V\left(t_{k}, y_{t_{k}}, j, x_{t_{k}}\right) \Delta t_{k+1} \\
E\left(\Delta V\left(t_{k+1}, y_{t_{k+1}}, j, x_{t_{k+1}}\right)-\left.E\left(\Delta V\left(t_{k+1}, y_{t_{k+1}}, j, x_{t_{t_{k+1}}}\right) \mid \mathbb{G}_{t_{t_{k}}}\right)\right|_{\mathbb{G}_{t_{k}}}\right)^{2}=E\left[\left[x_{t_{k}} \frac{\partial V}{\partial x} \sigma\left(j, t_{k}\right) \Delta B_{t_{k+1}}\right]^{2} \mid \mathbb{G}_{t_{k}}\right] \\
+E\left[\left[\int_{|z| \leq 1}\left[V\left(t_{k}, y_{t_{k}}, j, x_{t_{k}} e^{G(z, j)}\right)-V\left(t_{k}, y_{t_{k}}, j, x_{t_{k}}\right)\right] \bar{\psi}\left(j, d z, \Delta t_{k+1}\right)\right]^{2} \mid \mathbb{G}_{t_{k}}\right] \\
+E\left[\left[\int_{|z|>1}\left[V\left(t_{k}, y_{t_{k}}, j, x_{t_{k}} e^{H(z, j)}\right)-V\left(t_{k}, y_{t_{k}}, j, x_{t_{k}}\right)\right] \bar{\psi}\left(j, d z, \Delta t_{k+1}\right)\right]^{2} \mid \mathbb{G}_{t_{k}}\right] \\
+2 E\left[\left[x_{t_{k}} \frac{\partial V}{\partial x} \sigma\left(j, t_{k}\right) \Delta B_{t_{k+1}} \int_{|z| \leq 1}\left[V\left(t_{k}, y_{t_{k}}, j, x_{t_{k}} e^{G(z, j)}\right)-V\left(t_{k}, y_{t_{k}}, j, x_{t_{k}}\right)\right] \bar{\psi}\left(j, d z, \Delta t_{k+1}\right)\right] \mid \mathbb{G}_{t_{k}}\right] \\
+2 E\left[\left[x_{t_{k}} \frac{\partial V}{\partial x} \sigma\left(j, t_{k}\right) \Delta B_{t_{k+1}} \int_{\mid k>1}\left[V\left(t_{k}, y_{t_{k}}, j, x_{t_{k}} e^{H(z, j)}\right)-V\left(t_{k}, y_{t_{k}}, j, x_{t_{k}}\right)\right] \bar{\psi}\left(j, d z, \Delta t_{k+1}\right)\right] \mid \mathbb{G}_{t_{k}}\right] .
\end{gathered}
$$

$\psi$ and $B$ are independent martingales. Hence, the products involving both have zero expectations. We also note that products involving the compensated Poisson measure $\psi$ for large and small jumps vanish as they never jump, simultaneously. From Ito isometry, (12) becomes:

$$
\begin{gathered}
E\left(\Delta V\left(t_{k+1}, y_{t_{k+1}}, j, x_{t_{k+1}}\right)-E\left(\Delta V\left(t_{k+1}, y_{t_{k+1}}, j, x_{t_{k+1}}\right) \mid \mathbb{G}_{t_{k}}\right) \mid \mathbb{G}_{t_{k}}\right)^{2}=\left[x_{t_{k}} \frac{\partial V}{\partial x} \sigma\left(j, t_{k}\right)\right]^{2} \Delta t_{k+1} \\
+\quad \int_{|z| \leq 1}\left[V\left(t_{k}, y_{t_{k}}, j, x_{t_{k}} e^{G(z, j)}\right)-V\left(t_{k}, y_{t_{k}}, j, x_{t_{k}}\right)\right]^{2} v(j, d z) \Delta t_{k+1} \\
+\int_{k \mid>1>1}\left[V\left(t_{k}, y_{t_{k}}, j, x_{t_{k}} e^{H(z, j)}\right)-V\left(t_{k}, y_{t_{k}}, j, x_{t_{k}}\right)\right]^{2} v(j, d z) \Delta t_{k+1}
\end{gathered}
$$

This establishes the results.

The following remark describes the jump integral estimation problem.

Remark 3.1. (8) and (9) form the building blocks of the estimation procedure that is utilized to estimate the drift and diffusion coefficients. It is therefore possible that due to round off, discretization and computational errors, have negative values. Hence, it is critically important to choose an efficient numerical estimation methods of the Levy integrals. We chose to estimate Levy integrals via Monte Carlo integration method. We first note that compound Poisson processes have independent and identically distributed (iid) jump sizes. Hence, jumps sizes of Levy integrals are iid. We can apply the following Monte Carlo estimation scheme defined in Robert \& Casella (2013):

$$
\int_{z \in \square} g(z) v(j, d z)=E^{v(j,)}[g(z)] \approx \frac{1}{n(j)} \sum_{k=1}^{n(j)} g\left(z_{k}\right),
$$


where $g$ is a $v\left(j\right.$,)-integrable real valued function, and $\left(z_{i}\right)_{i=1}^{n(j)}$ is an iid sample of Levy jump sizes when the market is in state $\theta_{t}=j . n(j)$ denotes the number of Levy jump corresponding to the $j$ - th regime.

In the following Lemma, we present a particular case of interest along with an explicit formula for parameter estimates and a recursive formula for price simulation updates.

Lemma 3.2. (i) If $\mathrm{H}(\mathrm{z}, \mathrm{j})=\mathrm{G}(\mathrm{z}, \mathrm{j})=\mathrm{z}, \forall \mathrm{z} \in \mathbb{R}, \forall \mathrm{j} \in \mathrm{E}$ and $\mathrm{V}(\mathrm{t}, \mathrm{yt}, \mathrm{j}, \mathrm{x}(\mathrm{t}))=\ln (\mathrm{x}(\mathrm{t}))$, then the transformed stochastic Levy type differential equation, and the conditional expectations of Euler-Maruyama type discretization scheme in Lemma 3.1 reduce to:

$$
\begin{gathered}
d V\left(s, y_{s}, \theta_{s}, x_{s}\right)=\mathcal{L} V\left(s, y_{s^{-}}, \theta_{s^{-}}, x_{s^{-}}\right)+\sigma\left(\theta_{s^{-}}, s\right) x_{s^{-}} \frac{\partial V}{\partial x} d B_{s} \\
+\int_{|z| \leq 1}\left[V\left(s, y_{s^{-}}, \theta_{s^{-}}, x_{s^{-}} e^{G(z, j)}\right)-V\left(s, y_{s^{-}}, \theta_{s^{-}}, x_{s^{-}}\right)\right] \bar{\psi}(j, d z, d s) \\
+\int_{|z|>1}\left[V\left(s, y_{s^{-}}, \theta_{s^{-}}, x_{s^{-}} e^{H(z, j)}\right)-V\left(s, y_{s^{-}}, \theta_{s^{-}}, x_{s^{-}}\right)\right] \bar{\psi}(j, d z, d s) \\
E\left[\Delta \ln \left(x_{t_{k+1}}\right) \mid \mathcal{G}_{t_{k}}\right]=\left[\int_{|z|>1} z v(j, d z)+\mu\left(j, t_{k}\right)\right] \Delta t_{k+1} \\
E\left[\left(\Delta \ln \left(x_{t_{k+1}}\right)-E\left(\Delta \ln \left(x_{t_{k+1}}\right) \mid \mathcal{G}_{t_{k}}\right)\right) \mid \mathbb{G}_{t_{k}}\right]^{2}=\sigma^{2}\left(j, t_{k}\right) \Delta t_{k+1}+\int_{z \in \square} z^{2} v(j, d z) \Delta t_{k+1} .
\end{gathered}
$$

(ii)

At time $t_{k}$, we consider the sub-partition $P_{M_{n}, m_{k}}^{n, k}=\left\{t_{n-m_{k}}, t_{n-m_{k}+1}, \ldots, t_{n-1}\right\}$ of $P_{M_{n}}^{n} \quad$ consisting of the past $m_{k}$ consecutive data values of the price process $x_{t}$. We assume $\Delta t_{k}=\Delta t, \mu(j, t)=\mu(j)$ and $\sigma(j, t)=\sigma(j)$. We denote $\hat{\mu}_{t_{k}, m_{k}}^{j}$ and $\hat{\sigma}_{t_{k}, m_{k}}^{j}$ the estimates of $\mu(j)$ and $\sigma(j)$ relative to the sub partition $P_{M_{n}, m_{k}}^{n, k}$, respectively. Explicit formulas for $\hat{\mu}_{t_{k}, m_{k}}^{j}$ and $\hat{\sigma}_{t_{k}, m_{k}}^{j}$ can be expressed as follows:

$$
\begin{gathered}
\hat{\mu}_{t_{k}, m_{k}}=\frac{1}{m_{k} \Delta t} \sum_{i=k-m_{k}}^{k-1} E\left(\Delta \ln \left(x_{t_{i}}\right) \mid \mathbb{G}_{t_{i-1}}\right)-\frac{1}{n(j)} \sum_{k=1}^{n(j)} z_{k} 1_{\left|z_{k}\right|>1} \\
\left(\hat{\sigma}_{t_{k}, m_{k}}^{j}\right)^{2}=\frac{1}{m_{k}-1} \sum_{i=k-m_{k}}^{k-1} E\left(\Delta \ln \left(x_{t_{i}}\right)-E\left(\Delta \ln \left(x_{t_{i}}\right) \mid \mathbb{G}_{t_{i-1}}\right) \mid \mathbb{G}_{t_{i-1}}\right)^{2}-\frac{m_{k}}{m_{k}-1} \sum_{i=n-m_{k}}^{k-1} z_{i} \Delta t_{k}
\end{gathered}
$$

(iii)

We denote $\hat{x}_{t_{k}}=E\left[x_{t_{k}} \mid \mathbb{G}_{t_{k-1}}\right]$ the estimated conditional mean asset price. The following recurrence relation holds:

$$
\text { 资 }=x_{t_{k}} \exp \left[\text { 旗, } m_{k} \Delta t_{k+1}+\frac{1}{2}\left(\sigma_{t_{k}, m_{k}}^{j}\right)^{2} \Delta t_{k+1}+\frac{1}{n(j)} \sum_{l=1}^{n(j)}\left[e^{z_{l}}-1-z_{l} 1_{\left|z_{l}\right| \leq 1}\right]\right] .
$$


Proof. Under the assumption $H(z, j)=G(z, j)=z, \forall z \in \mathbb{R}, \forall j \in E$, using $V\left(t, y_{t}, j, x(t)\right)=\ln (x(t))$, and applying Lemma $3.1,(8)$ and (9) reduce to (16) and (17), respectively. (ii) is a direct consequence of part (i). Summing up (18) and (19) over the sub partition $P_{M_{n}, m_{k}}^{n, k}$ we obtain:

$$
\begin{gathered}
\sum_{i=k-m_{k}}^{k-1} E\left[\Delta \ln \left(x_{t_{k}}\right) \mid \mathcal{G}_{t_{k-1}}\right]=m_{k}\left[\frac{1}{n(j)} \sum_{k=1}^{n(j)} z_{k} 1_{\left|z_{k}\right|>1}+m_{k} \mu(j)\right] \Delta t \\
\sum_{i=k-m_{k}}^{k-1} E\left[\left(\Delta \ln \left(x_{t_{k}}\right)-E\left(\Delta \ln \left(x_{t_{k}}\right) \mid \mathcal{G}_{t_{k-1}}\right)\right) \mid \mathbb{G}_{t_{k-1}}\right]^{2}=m_{k} \sigma^{2}(j) \Delta t+m_{k} \int_{z \in \square} z^{2} v(j, d z) \Delta t
\end{gathered}
$$

Solutions of algebraic equations in (21) and (22) establish (18) and (19), respectively. For (iii), we consider $n \in I(1, M$ ) and $t_{n-1}, t_{n}$, points of the partition $P_{M}$ such that $T_{k-1}<t_{n-1}<t_{n}<T_{k}$ where $\theta_{T k-1}=j$ for some $k \in I(1, \infty)$. By Levy Kintchine formula Øksendal \& Sulem (2005), we have:

$$
\begin{gathered}
E\left[x_{t_{n}} \mid \mathcal{G}_{t_{n-1}}\right]=E\left[x_{t_{n-1}} \exp \left[\int_{t_{n-1}}^{t_{n}} d L_{s}^{j}\right] \mid \mathcal{G}_{t_{n-1}}\right] \\
=E\left[x_{t_{n-1}} \exp \left[\mu(j) \Delta t+\sigma(j) \Delta B_{n}+\int_{t_{n-1}}^{t_{n}} \int_{|z|>1} z \psi(j, d z, d s)+\int_{t_{n-1}}^{t_{n}} \int_{|z| \leq 1} z \bar{\psi}(j, d z, d t)\right] \mid \mathcal{G}_{t_{n-1}}\right] \\
\left.x_{t_{n}}=x_{t_{n-1}} \exp \left[\partial \operatorname{rec}^{2} j\right) \Delta t+\frac{1}{2} \sigma^{2}(j) \Delta t+\frac{\Delta t}{n(j)} \sum_{k=1}^{n(j)}\left[e^{z_{k}}-1-z_{k} 1_{\left|z_{k}\right|<1}\right]\right]
\end{gathered}
$$

Hence, at each time step $t_{n}$, the simulated conditional mean observation is computed recursively as follows:

$$
\text { 资 } \left.=x_{t_{n-1}} \exp [\text { 弥 } j) \Delta t+\frac{1}{2} \sigma^{2}(j) \Delta t+\frac{\Delta t}{n(j)} \sum_{k=1}^{n(j)}\left[e^{z_{k}}-1-z_{k} 1_{\left|z_{k}\right| k}\right]\right],
$$

where $n(j)$ is the size of the data when the market is in regime $\mathrm{j}$, hence proving the lemma.

\subsection{Parameter Estimation for Three Real Data.}

We assume the regime switching times observable. Although semi Markov jumps $\beta$, are not expected to stand out by their size, large jumps have empirically been associated with local structural changes through clustering Bulla (2006) and will therefore be chosen as jump times. These jump times could be used to estimate the sojourn time parameters of the semi Markov process; however, we focus on estimating the price jump distribution parameters and the parameters of the Levy distributions in between jumps. The IBM, Bank of America Corporation and BNP Paris Bas data were collected over a period of 20 years and six months (daily except for the weekends and market holidays) from January 2nd 1994 to July 11th 2014. Structural changes were identified in each data set and the corresponding jumps were considered semi Markov market price jumps. Based on Lemma 3.2, we estimate the parameters of model (4) between jumps using the LLGMM algorithm developed in Otunuga (2014) and Otunuga et al (2017). The fit of the LLGMM is presented by the first columns of Figures 1, 2 and 3. Another feature of the LLGMM mentioned in Otunuga (2014) and Otunuga et al (2017), is the more obvious randomness in the volatility as opposed to that of Garch $(1,1)$. The same conclusion is reached in the context of semi Markov exponential Levy asset prices as shown in the second columns of Figures 1, 2 and 3. As noted in Remark 3.1, jumps are independent and so are log jumps. Estimation of the parameters of the distribution of $\beta$ could be performed by maximum likelihood. 

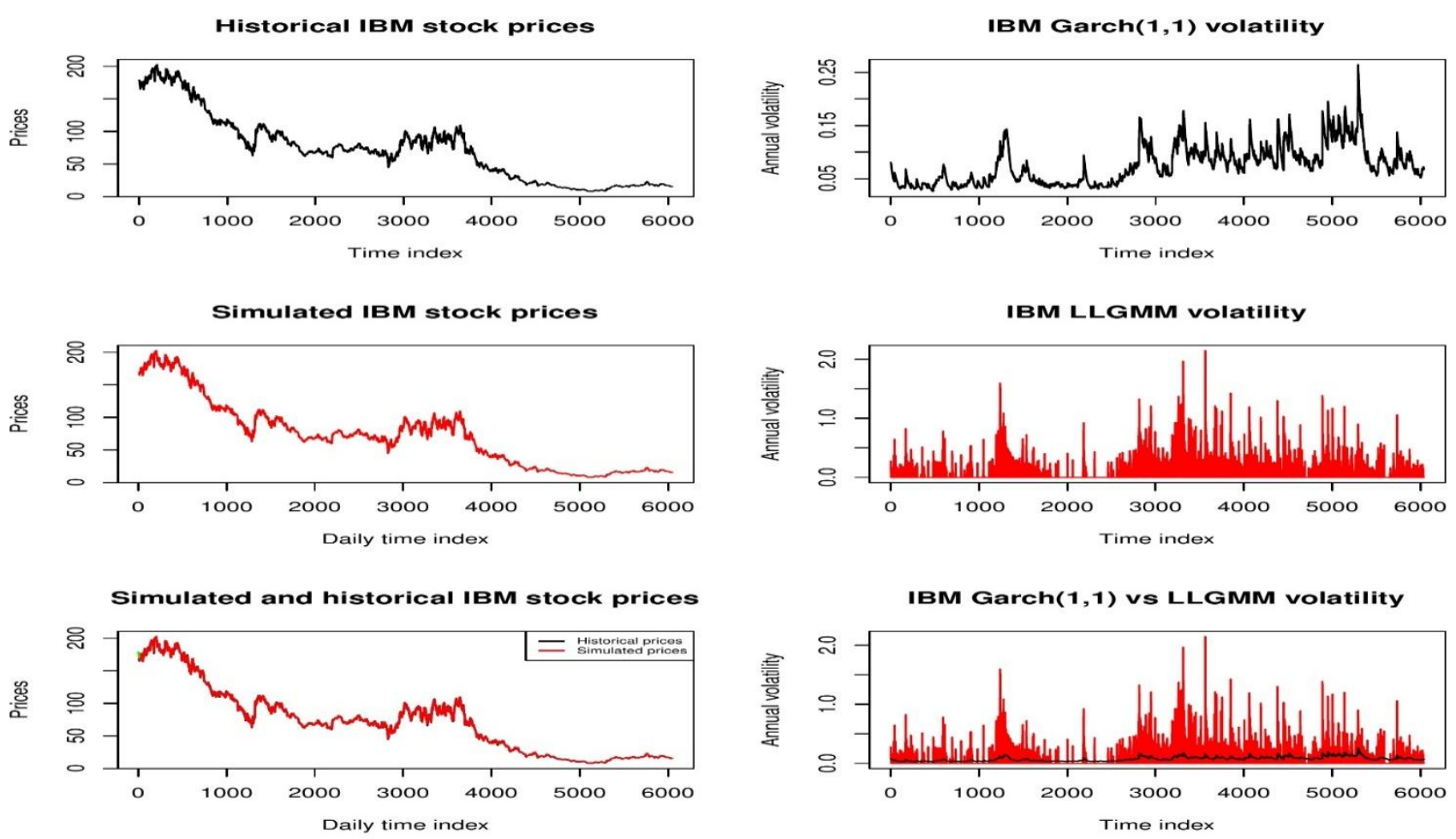

Figure 1. The First Column Exhibits the Fit of the LLGMM Simulated IBM Stock Prices against Historical Prices. The Second Column Illustrates a Comparison of LLGMM and Garch $(1,1)$ Annual Volatilities.
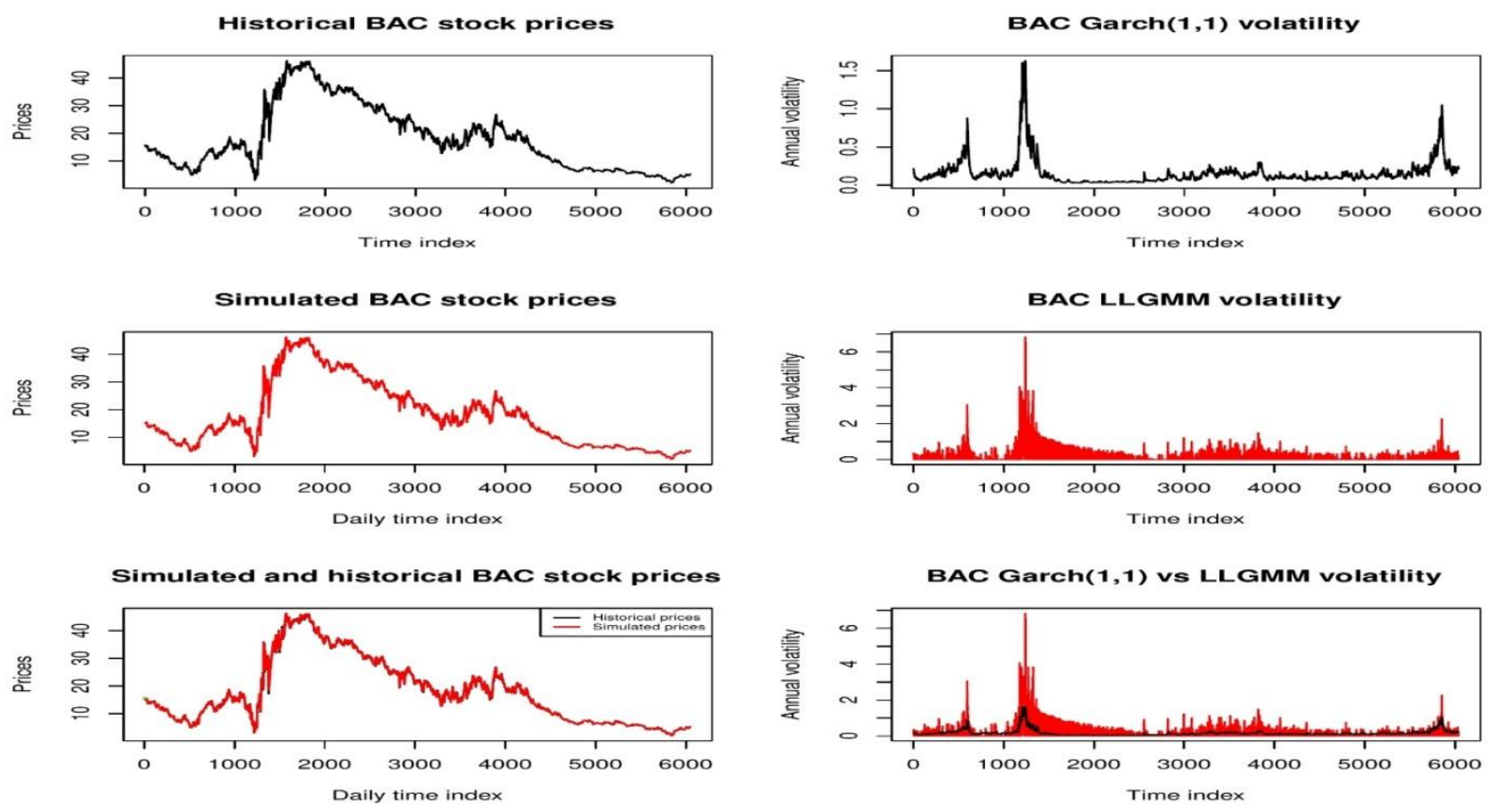

Figure 2. The First Column Shows the Fit for the LLGMM simulated BAC Stock Prices Against Historical Prices. The Second Column Exhibits the Performance of the LLGMM and Garch (1,1) Annual Volatilities. 

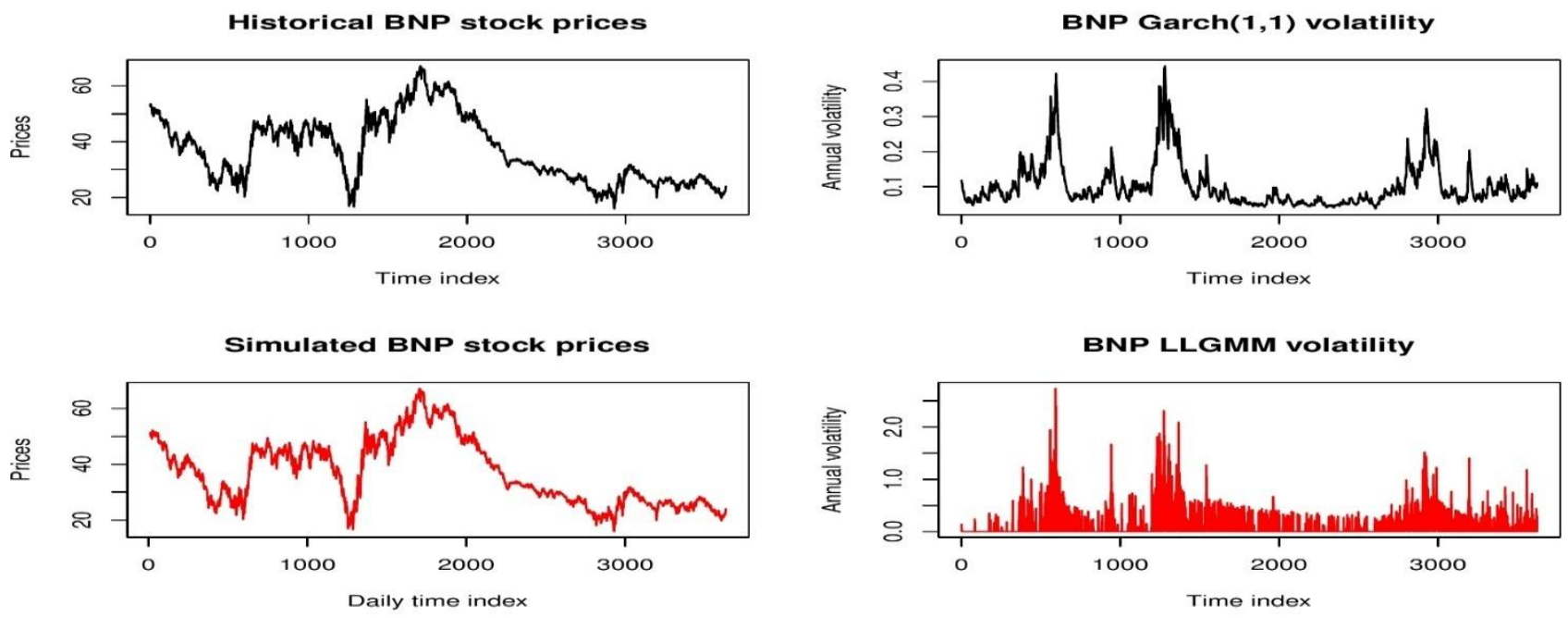

Simulated and historical BNP stock prices

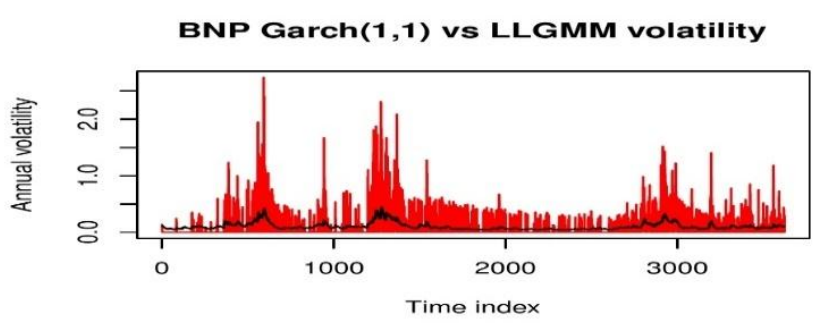

Figure 3.The First Column Exhibits the Fit of the LLGMM stock of Bank National de Paris (BNP) against Historical

Prices. The Second Column Illustrates A Comparison of LLGMM and Garch $(1,1)$ Annual Volatilities.

Table 1. IBM, BAC and BNP Data Parameter Estimates and Simulation Results IBM: Overview of a few LLGMM Parameter Estimates and Simulated Values.

\begin{tabular}{ccccccc}
\hline Index: $t_{k}$ & Data: $y_{t k}$ & Estimate: $\hat{y}_{k}$ & Error: $\left|y_{t k}-\hat{y}_{k}\right|$ & Volatility: $s_{k}$ & Drift: $(j)$ & Sample: ${ }_{k}:$ \\
\hline 11 & 167.73 & 169.19 & 0.70 & 0.000033 & -0.013 & 2 \\
12 & 169.72 & 168.82 & 0.90 & 0.000404 & -0.002 & 2 \\
13 & 164.90 & 166.98 & 2.08 & 0.000434 & -0.011 & 3 \\
14 & 165.45 & 165.71 & 0.27 & 0.000257 & -0.008 & 5 \\
15 & 167.19 & 166.87 & 0.32 & 0.000026 & 0.007 & 2 \\
16 & 169.02 & 168.68 & 0.35 & 0.000000 & 0.011 & 2 \\
17 & 169.35 & 168.97 & 0.38 & 0.000240 & 0.002 & 6 \\
18 & 169.55 & 169.77 & 0.22 & 0.000029 & 0.005 & 3 \\
19 & 168.03 & 169.12 & 1.08 & 0.000052 & -0.004 & 2 \\
6043 & 15.73 & 15.60 & 0.12 & 0.000259 & 0.009 & 2 \\
6044 & 15.59 & 15.59 & 0.00 & 0.000170 & -0.001 & 296 \\
6045 & 15.65 & 15.64 & 0.01 & 0.000156 & 0.003 & 4 \\
6046 & 15.80 & 15.75 & 0.06 & 0.000019 & 0.007 & 2 \\
6047 & 15.71 & 15.72 & 0.01 & 0.000116 & -0.002 & 100 \\
6048 & 15.75 & 15.75 & 0.00 & 0.000136 & 0.002 & 14 \\
6049 & 15.57 & 15.67 & 0.10 & 0.000049 & -0.005 & 3 \\
6050 & 15.43 & 15.51 & 0.08 & 0.000003 & -0.010 & 2 \\
\hline
\end{tabular}

BAC: Overview of a few LLGMM Parameter Estimates and Simulated Values. 


\begin{tabular}{ccccccc}
\hline Index: $t_{k}$ & Data: $y_{t k}$ & Estimate: $\hat{y}_{k}$ & Error: $\left|y_{t k}-\hat{y}_{k}\right|$ & Volatility: $s_{k}$ & Drift: $(j)$ & Sample: ${ }_{k}:$ \\
\hline 11 & 14.98 & 15.35 & 0.22 & 0.000427 & -0.018 & 2 \\
12 & 15.04 & 15.13 & 0.09 & 0.000684 & -0.015 & 2 \\
13 & 14.98 & 14.97 & 0.01 & 0.000380 & -0.011 & 3 \\
14 & 15.05 & 14.99 & 0.06 & 0.000023 & 0.002 & 3 \\
15 & 15.05 & 15.03 & 0.02 & 0.000011 & 0.002 & 2 \\
16 & 15.35 & 15.18 & 0.17 & 0.000202 & 0.010 & 2 \\
17 & 15.37 & 15.34 & 0.03 & 0.000177 & 0.011 & 2 \\
18 & 15.35 & 15.35 & 0.01 & 0.000127 & 0.000 & 15 \\
19 & 15.23 & 15.27 & 0.05 & 0.000025 & -0.005 & 2 \\
6043 & 5.10 & 5.04 & 0.05 & 0.000000 & 0.029 & 2 \\
6044 & 5.14 & 5.14 & 0.00 & 0.000207 & 0.019 & 2 \\
6045 & 5.24 & 5.24 & 0.00 & 0.000104 & 0.019 & 3 \\
6046 & 5.24 & 5.24 & 0.00 & 0.000650 & 0.000 & 4819 \\
6047 & 5.21 & 5.23 & 0.02 & 0.000015 & -0.003 & 2 \\
6048 & 5.33 & 5.31 & 0.02 & 0.000194 & 0.015 & 7 \\
6049 & 5.44 & 5.42 & 0.02 & 0.000000 & 0.022 & 2 \\
6050 & 5.40 & 5.42 & 0.02 & 0.000324 & -0.001 & 2156 \\
\hline
\end{tabular}

BNP: Overview of a few LLGMM Parameter Estimates and Simulated Values.

\begin{tabular}{ccccccc}
\hline Index: $t_{k}$ & Data: $y_{t k}$ & Estimate: $\hat{y}_{k}$ & Error: $\left|y_{t k}-\hat{y}_{k}\right|$ & Volatility: $s_{k}$ & Drift: $(j)$ & Sample: ${ }_{k}$ \\
\hline 11 & 51.40 & 51.60 & 0.09 & 0.000078 & -0.008 & 3 \\
12 & 50.89 & 51.01 & 0.12 & 0.000014 & -0.012 & 3 \\
13 & 49.89 & 50.28 & 0.39 & 0.000049 & -0.015 & 2 \\
14 & 51.00 & 50.36 & 0.64 & 0.000883 & 0.001 & 2 \\
15 & 50.22 & 50.22 & 0.00 & 0.000155 & -0.003 & 14 \\
16 & 49.99 & 49.99 & 0.00 & 0.000111 & -0.005 & 14 \\
17 & 50.16 & 50.08 & 0.08 & 0.000252 & 0.001 & 4 \\
18 & 50.38 & 50.30 & 0.08 & 0.000000 & 0.004 & 2 \\
19 & 50.49 & 50.49 & 0.00 & 0.000001 & 0.003 & 3 \\
3626 & 21.93 & 22.05 & 0.11 & 0.000202 & -0.008 & 2 \\
3627 & 21.73 & 21.87 & 0.14 & 0.000102 & -0.008 & 3 \\
3628 & 22.56 & 22.19 & 0.37 & 0.001114 & 0.014 & 2 \\
3629 & 22.42 & 22.36 & 0.06 & 0.000696 & 0.007 & 3 \\
3630 & 22.14 & 22.16 & 0.01 & 0.000017 & -0.009 & 2 \\
3631 & 22.92 & 22.46 & 0.46 & 0.000697 & 0.013 & 4 \\
3632 & 23.43 & 23.11 & 0.32 & 0.000082 & 0.028 & 2 \\
3633 & 24.10 & 23.78 & 0.32 & 0.000041 & 0.028 & 3 \\
\hline
\end{tabular}

\section{Option Pricing}

We develop option pricing based on Carr and Madan method and the FST method. We first recall the following definitions from Assonken \& G.S. (2015) useful in the remainder of this section:

Definition 4.1. Let $\mathrm{S}$ denotes a continuous function defined on $\mathbb{R}^{+} \times \mathbb{R}^{+}$into $\mathbb{R}$ representing the payoff of a contingent claim; $\mathrm{Q}$ is a risk neutral probability measure of the price process $\mathrm{x}$ defined by (4) with respect to the historical probability measure $\mathrm{P} ; \mathrm{K}$ is a nonnegative real number denoting the strike price of a derivative contract with maturity $\mathrm{T}$;

$x_{T}$ denotes the asset price value at maturity; $\mathrm{C}$ is the Q-risk neutral option price function defined on $[0, \mathrm{~T}] \times \mathbb{R}^{+} \times \mathbb{R}^{+}$ $\times[0, \mathrm{~T}] \times \mathrm{E} \times \mathbb{R}^{+}$into $\mathbb{R}^{+}$and $\mathrm{V}$ denotes the discounted option price process defined by

$$
V\left(t, T, K, y_{t}, \theta_{t}, x_{t}\right)=e^{-\int_{0}^{t} r_{u} d u} C\left(t, T, K, y_{t}, \theta_{t}, x_{t}\right)
$$


The Fourier transform and the inverse Fourier transform of an integrable function $f$, are interchangeably denoted $\mathcal{F}(f)$ or $\quad \hat{f}$ and $\mathcal{F}^{-1}(f)$ or $\breve{f}$, respectively. Let $N(t, A, B)$ be a stochastic process defined on $[0, T] \times \mathrm{B}(\mathbb{R}) \times P\left(E^{2}\right)$ into $[0, \infty)$ as:

and $N(t, A, B)$ stands for the number of regime switches in $B$ with corresponding log price jumps $\ln \left(\beta_{n}\right) \in A$ by time $t$. The compensators $\gamma(t, A,\{(i, j)\})=\int_{0}^{t} \int_{z \in A} b(z \mid i, j) \lambda_{i, j}\left(y_{s}\right) d z d s$ of $N(t, A,\{(i, j)\})$ are derived in Assonken \& G.S. (2015).

$$
\left.N(t, A, B)=\sum_{n \geq 1} 1_{\left(t \geq T_{n}, \ln \left(\beta_{n}\right) \in A,\left(\theta_{n-1}, \theta_{n}\right) \in B\right.}\right)
$$

We model asset prices with the semi Markov switching exponential Levy process in (5), where $L^{\theta}$ defined in (2) is based on $H(z, j)=G(z, j)=z$. Options are priced based on the risk neutral theory. The martingale probability measure chosen for pricing purpose is the conditional minimum entropy martingale measure (CMEMM) $P^{\alpha \star}$ with density process expressed in Assonken \& G.S. (2015) as the following Esscher transform:

$$
\left.\frac{d \bar{P}^{\alpha^{\star}}}{d P}\right|_{\mathbb{H}_{T} \vee \mathbb{L}_{t}}=\left[\prod_{i=1}^{n} \frac{e^{\alpha_{i}^{\star} \beta_{i}}}{E\left(e^{\alpha_{i}^{\star} \beta_{i}} \mid \theta_{i}, \theta_{i-1}\right)}\right] \frac{e^{\int_{0}^{t} \alpha_{s}^{\star} d L_{s}}}{E\left(e^{\int_{0}^{t} \alpha_{s}^{\star} d L_{s}} \mid \mathbb{H}_{T}\right)}, \forall t \in\left[T_{n}, T_{n+1}\right) .
$$

From Assonken \& G.S. (2015), for $\forall t \in\left[T_{n}, T_{n+1}\right), \forall n \in I(1, \infty)$, the risk neutral conditions satisfied by the Esscher parameter process $\left(\alpha_{t}^{\star}\right)_{t \in[0, T]}$, are as follows:

$$
\left\{\begin{array}{l}
\mu\left(\theta_{n}\right)+\alpha_{t}^{\star} \sigma^{2}\left(\theta_{n}\right)+\int_{|z| \leq 1}\left[G\left(z, \theta_{n}\right) e^{\alpha_{t}^{*} G\left(z, \theta_{n}\right)}-G\left(z, \theta_{n}\right)\right] v\left(\theta_{n}, d z\right) \\
+\int_{|z|>1} H\left(z, \theta_{n}\right)\left(e^{\alpha_{t}^{\star} H\left(z, \theta_{n}\right)}-1\right) v\left(\theta_{n}, d z\right)=r(t), \forall t \in\left(T_{n}, T_{n+1}\right) \\
{\left[E^{p^{\alpha^{\star}}}\left[\beta_{n} \mid \theta_{n}, \theta_{n-1}\right]-1\right]=\left[E\left[\frac{\beta_{n} e^{\alpha_{t}^{\star} \beta_{n}}}{E\left[e^{\alpha_{t}^{*} \beta_{n}} \mid \theta_{n}, \theta_{n-1}\right]} \mid \theta_{n}, \theta_{n-1}\right]-1\right]=0 \text { if } t=T_{n} .}
\end{array}\right.
$$

The risk neutral pricing formula for European call options is described by:

$$
\begin{gathered}
C\left(t, T, K, y_{t}, j, x_{t}\right)=E^{\bar{P}^{\alpha}}\left(e^{-\int_{t}^{T} r_{s} d s}\left(x_{T}-K\right)^{+} \mid y_{t}, \theta_{t}=j, x_{t}\right) \\
=E^{\bar{P}^{\alpha}}\left(e^{-\int_{t}^{T} r_{s} d s}\left(e^{\ln \left(x_{T}\right)}-e^{k}\right)^{+} \mid y_{t}, \theta_{t}=j, x_{t}\right) \\
=E^{\bar{P}^{\alpha}}\left(e^{k-\int_{t}^{T} r_{s} d s}\left(e^{k\left(\frac{\ln \left(x_{T}\right)}{k}-1\right)}-1\right)^{+} \mid y_{t}, \theta_{t}=j, x_{t}\right),
\end{gathered}
$$

where $k=\ln (K)$. Given the known closed form expression of the characteristic function of $\log$ prices Assonken \& G.S. (2015), one can apply Carr and Madan formula Carr \& Madan (1999):

$$
\mathrm{Y}\left(t, T, u, y_{0}, j, x_{0}\right)=\frac{e^{-\int_{t}^{T} r_{s} d s}}{(\alpha+i u)(1+\alpha+i u)} \Psi\left(u-i(1+\alpha), t, y_{0}, j, x_{0}\right),
$$


where $\Upsilon$ is the characteristic function of the modified option price

$$
\tilde{C}\left(t, T, k, y_{t}, j, x_{t}\right)=e^{\alpha k} E^{\bar{P}^{\alpha}}\left[e^{-\int_{t}^{T} r_{s} d s}\left(e^{\ln \left(x_{T}\right)}-e^{k}\right)^{+} \mid y_{t}, \theta_{t}=j, x_{t}\right]
$$

is the characteristics function of the log prices developed in Assonken \& G.S. (2015):

$$
\begin{gathered}
\Psi(t, T, u, x, j, y)=E\left[e^{i u \ln \left(x_{t}\right)} \mid \theta_{0}=j, y_{0}=y, x_{0}=x\right] \\
=\exp \left(i u \ln \left(x_{t}\right)\right)\left\langle\exp \left(\int_{y}^{t+y} M(u, \eta) d \eta\right) e_{j}, 1\right\rangle,
\end{gathered}
$$

and $M$ is an $m \times m$ matrix function with components $\quad\left(M_{p q}\right)_{1 \leq p, q \leq m} \quad$ are defined by:

$$
M_{q p}(u, y)=\left\{\begin{array}{l}
i u \mu(q)-\frac{1}{2} \sigma^{2}(q) u^{2}+\int_{|z| \leq 1}\left[e^{i u G(z, q)}-1-i u G(z, q)\right] v(q, d z) \\
+\int_{\mid z>>1}\left[e^{i u H(z, q)}-1\right] v(q, d z)+\lambda_{q, q}(y), \text { if } p=q \\
\lambda_{q, p}(y) \int_{z \in \mathbb{I}} e^{i u r} \bar{b}(z \mid q, p) d z, \text { otherwise. }
\end{array}\right.
$$

$M$ is assumed to satisfies the Lie bracket condition Magnus (1954)

$$
\left[M\left(u, t_{1}\right), M\left(u, t_{2}\right)\right]=0, \forall t_{1}, t_{2} \in \mathbb{R}_{+} .
$$

$\mu$ satisfies the martingale condition in (25). We next explore the effects of parameters on option prices and implied volatilities. By developing a closed form solution to a PIDE which will allow us to apply the FST algorithm, hence paving the way for the pricing of exotic and American options R Jackson (2009) in the context of asset price model (4). The option price is the inverse Fourier transform of $\Upsilon$ :

$$
C\left(t, T, k, y_{t}, j, x_{t}\right)=\frac{e^{-\alpha k}}{2 \pi} \int e^{-i u k} \mathrm{Y}(t, T, u, y, j, x) d u .
$$

The Fourier time stepping method from R Jackson (2009), is an option pricing method of vanilla and exotic option contracts based on the inverse Fourier transform. The FST has been used to price options when the market is subjected to Markov regime changes, Jackson et al. (2007); Momeya (2012); R Jackson (2009). We investigate an application of the FST method to semi Markov regimes with jumps at regime changes. We assume in this subsection that the asset price process in (5) is defined under a $(P, \mathbb{H} \vee \mathbb{L})$ equivalent martingale measure $Q$.

Lemma 4.1. Let $\mathrm{S}$ be a random variable representing the payoff of a general European style contingent claim with maturity $\mathrm{T}$ and strike price $\mathrm{K}$ in Definition 4.1; let $\mathrm{Q}$ and be the risk neutral measure and the infinitesimal generator defined in Definitions 4.1 and 6. Let $\mathrm{C}$ be the Q-risk neutral option price of a contingent claim.

(i)

Then, for $\theta_{s}^{-}=j$, the $Q$-risk neutral option price $C$ of a European contingent claim with maturity $T$, strike price $K$ and payoff $S$ satisfies the following PIDE: 


$$
\left\{\begin{array}{l}
\mathcal{L} V\left(s, T, K, y_{s^{-}}, j, x_{s^{-}}\right)=0 \\
V\left(T, T, K, y_{T}, \theta_{T}, x_{T}\right)=e^{\int_{0}^{T} r_{u} d u} S\left(x_{T}, K\right)
\end{array}\right.
$$

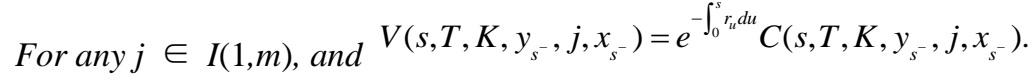

(ii)

The vector solution $\mathbf{C}(s, T, K y \ngtr \neq(C s(T, K, y, x 1, C) s, T(K, y, \quad x, 2, C) s, T . K y m x)$, has Fourier Transform (with respect to $\ln (\mathrm{x})$ ):

$$
\begin{aligned}
& \mathbf{C}(s, T, K, y, w)=[\text { 瓷 } s, T, K, y, 1, w), C(s, T, K, y, 2, w), \ldots, \mathcal{C}(s, Y, K, y, m, w)] \\
& =\exp \left[\int_{y}^{T-t+y} M(u, s) d s\right] .[\hat{S}(w) \mathbf{1}],
\end{aligned}
$$

and the Fourier transform of option prices in individual regimes are:

$$
\text { 抙 } s, T, K, y, j, w)=\left\langle\exp \left[\int_{y}^{T-t+y} M(u, s) d s\right] \cdot\left[S(w) \cdot e_{j}\right], \mathbf{1}\right\rangle .
$$

Proof. (i) is a direct consequence of the PIDE derived in Assonken \& G.S. (2015) with $\mu, H$ and $G$ replaced by $\int_{k \mid \leqslant \leq 1}\left[e^{G(z, j)}-1-G(z, j)\right] v(j, d z)+\frac{1}{2} \sigma^{2}(j), e^{G(z, j)}-1, e^{H(z, j)}-1$ and $e^{H(z, j)}-1$. We prove (ii) using properties of the Fourier transform. Using the change of variable $x=\ln (x)$, assuming $S, C \in \mathrm{L}^{1}(\Omega, \mathrm{G}, Q)$ with respect to the first and the sixth variables, respectively, and using the properties :

$$
\left.\mathcal{F}\left(\frac{\partial^{n}}{\partial \bar{x}^{n}} C\right)(t, T, K, y, j, w)=(i w)^{n} \text { 瓷 } t, T, K, y, j, w\right) \text { and } \mathcal{F}\left(C\left(t, T, K, y, j, x e^{z}\right)\right)(w)=e^{i w z} C(t, T, K, y, j, w) \text {, along with linearity of the Fourier }
$$
transform lead to:

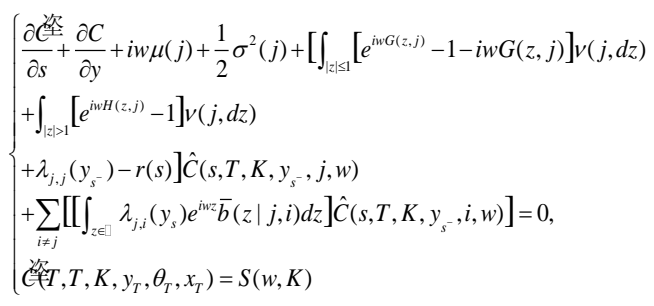

In vector form, (36) becomes:

$$
\left\{\begin{array}{l}
\frac{\partial \mathbf{C}}{\partial s}+\frac{\partial \mathbf{C}}{\partial y}+M(w, y) \mathbf{C}(s, y)=0 \\
\mathbf{C}\left(T, T, K, y_{T}, w\right)=\hat{S}(w, K) . \mathbf{1}
\end{array}\right.
$$




$$
M_{p, q}(w, y)= \begin{cases}i w \mu(p)-\frac{1}{2} w^{2} \sigma^{2}(p)+\int_{|z| \leq 1}\left[e^{i w G(z, p)}-1-i w e^{G(z, p)}\right] v(p, d z) & \\ +\int_{|z|>1}\left[e^{i w H(z, p)}-1\right] v(p, d z)+\lambda_{p, p}(y)-r(p) & \text { If } p=q \\ \lambda_{q, p}(y) \int_{z \in \square} e^{i w z} \bar{b}(z \mid q, p) d z & \text { Otherwise, }\end{cases}
$$

With the matrix $(M(w, y))_{m \times m}$ defined by its elements:

which proves (ii). Such a system of PIDE does not in general admit classical solutions as many payoff functions or derivative instruments are continuous but not differentiable. The solutions considered for this type of PIDE are weak solutions in the sense of viscosity, which are proven to exist in Momeya (2012) in the Markov regime switching case. We assume in our case that the conditional intensity matrix is a piecewise constant function of the backward recurrence time. Hence, PIDE (32) has a unique viscosity solution and is solved in Assonken \& G.S. (2015) yielding the general solution:

hence,

$$
\begin{gathered}
\mathbf{C}(s, T, K, y, w)=\exp \left[\int_{y}^{T-t+y} M(w, s) d s\right] \cdot[\hat{S}(w, K) \mathbf{1}], \\
\text { 资 } s, T, K, y, j, w)=\left\langle\exp \left[\int_{y}^{T-t+y} M(w, s) d s\right] \cdot[S(w, K) . \mathbf{1}], e_{j}\right\rangle .
\end{gathered}
$$

Which proves (iii).

Remark 4.1. We note that for any $t_{2}>t_{1}$ we have:

$$
\left\{\begin{array}{l}
\mathbf{C}\left(t_{1}, T, K, y, w\right)=\exp \left[\int_{y}^{t_{2}-t_{1}+y} M(w, s) d s\right] \cdot \mathbf{C}\left(t_{2}, T, K, y_{2}, w\right) \\
\hat{C}\left(t_{1}, T, K, y, j, w\right)=\left\langle\exp \left[\int_{y}^{t_{2}-t_{1}+y} M(w, s) d s\right] \cdot \mathbf{C}\left(t_{2}, T, K, y_{2}, w\right), e_{j}\right\rangle .
\end{array}\right.
$$

Remark 4.2. We recall the discretization necessary for implementing the FST algorithm, R Jackson (2009). First partition the time space $[0, T]$ in $N$ subintervals with $P^{t, n}=\left(t_{n}\right)_{n=0}^{N}$. Discretization of the log stock price space $(-\infty, \infty)$ is done by approximating the log price domain by a bounded domain $\left[x_{\min }, x_{\max }\right]$ and set $P^{x, n}=\left(x_{n}\right)_{n=0}^{M}, x_{i}=x_{\text {min }}+i \Delta x$ where $\Delta x=\frac{x_{\max }-x_{\min }}{N}$ As noted by R Jackson (2009), it is sometimes preferred to discretize either $\ln \left(x / x_{0}\right)$ or $\ln (x / K)$ with $K$ the strike price of the option contract depending on whether the pricing is needed around the strike price or not. The frequency domain $\left[0, w_{\max }\right]$ is partitioned with $P^{w, N}=\left(w_{i}\right)_{i=0}^{\frac{N+1}{2}}, w_{i}=x_{\min }+i \Delta w=\frac{2 w_{\max }}{N}$ and $w_{\max }=\frac{1}{2 \Delta x}$, the Niquisdt critical frequency.

Next lemma describes the basic difference between option prices in semi Markov regimes and Markov regimes and shows that it boils down mainly to the difference in integrated conditional intensities.

Lemma 4.2. Let $M, M^{m}, C^{m}, \Upsilon^{m}$ and $\Psi^{m}$ be the matrix defined by (29), the matrix defined by (29) when $\left(\theta_{t}\right)_{t \in[, T]}$ is a Markov process with generator matrix components denoted $\left(\lambda_{i, j}^{m}\right)_{1 \leq i, j \leq m}$, Carr and Madan option price via FFT, the 
characteristic function of option prices and the characteristic function of spot prices in Markov switching market regimes.

$$
\begin{aligned}
& \frac{2 \pi}{e^{-a k}}\left(C\left(t, T, k, y_{t}, j, x_{t}\right)-C^{m}\left(t, T, k, j, x_{t}\right)\right) \\
& =\int_{\square} \frac{\exp \left[\left(i u\left(\ln \left(x_{t}\right)-u k\right)\right)-\int_{t}^{T} r_{s} d s\right]}{(\alpha+i u)(1+\alpha+i u)} \sum_{n=1}^{\infty}\left\langle\left[\left(\int_{y}^{(t y} M(u-i(1+\alpha), \eta) d \eta\right)^{n}-\left(t M^{m}(u-i(1+\alpha))\right)^{n}\right] e_{j}, 1\right\rangle d u
\end{aligned}
$$

Proof.

$$
\begin{aligned}
& \frac{2 \pi}{e^{-\alpha k}}\left(C\left(t, T, k, y_{t}, j, x_{t}\right)-C^{m}\left(t, T, k, j, x_{t}\right)\right) \\
& =\frac{e^{-\alpha k}}{2 \pi} \int_{\square} e^{-i u k} \mathrm{Y}(t, T, u, y, j, x) d u-\frac{e^{-\alpha k}}{2 \pi} \int_{\square} e^{-i u k} \mathrm{Y}^{m}(t, T, u, j, x) d u, \\
& =\int e^{-i u k}\left(\mathrm{Y}(t, T, u, y, j, x)-\mathrm{Y}^{m}(t, T, u, j, x)\right) d u \quad \text {, from (31), } \\
& =\int \frac{e^{-i u k-T_{1}^{T}, r_{d s}}}{(\alpha+i u)(1+\alpha+i u)}\left[\Psi\left(u-i(1+\alpha), t, y_{0}, j, x_{0}\right)-\Psi^{m}\left(u-i(1+\alpha), t, j, x_{0}\right)\right] d u, \text { from (27) } \\
& =\int_{\square} \frac{\exp \left[\left(i u\left(\ln \left(x_{t}\right)-u k\right)\right)-\int_{t}^{T} r_{s} d s\right]}{(\alpha+i u)(1+\alpha+i u)}\left\langle\left[\exp \left(\int_{y}^{1+y} M(u-i(1+\alpha), \eta) d \eta\right)\right.\right. \\
& \left.\left.-\exp \left(t M^{m}(u-i(1+\alpha))\right)\right] e_{j}, 1\right\rangle d u \text {, } \\
& \text {, from (28) } \\
& =\int_{\square} \frac{\exp \left[\left(i u\left(\ln \left(x_{t}\right)-u k\right)\right)-\int_{t}^{T} r_{s} d s\right]}{(\alpha+i u)(1+\alpha+i u)} \sum_{n=1}^{\infty}\left\langle\left[\left(\int_{y}^{t+y} M(u-i(1+\alpha), \eta) d \eta\right)^{n}\right.\right. \\
& \left.\left.-\left(t M^{m}(u-i(1+\alpha))\right)^{n}\right] e_{j}, \mathbf{1}\right\rangle d u
\end{aligned}
$$

\subsection{Effect of Parameters on Option Prices.}

Simulation of option prices is performed by computing the inverse Fourier transform of (27). We use Simpson rule of integration, with upper limit of integration in $w$ being $a$. Moreover, the frequency space is divided into $\mathrm{N}$ subintervals of equal lengths; the log strike $\mathrm{k}$ ranges from $-b$ to $b$ divided into $\mathrm{N}$ subintervals of equal lengths. Inverting the Fourier Transform of could be done quite efficiently by FFT or even by FRFT as suggested in Chourdakis (2004). Let's use the notation: $w_{j}=(j-1) \eta$ with $\eta^{\eta=\frac{a}{N}} \quad k_{u}=-b+\lambda(u-1)$ with $\lambda=\frac{2 b}{N}$. To match the Discrete Fourier Transform with the FFT requires one to impose the condition $\lambda \eta=\frac{2 a b}{N}$ :

$$
C\left(t, T, k_{u}, y_{t}, j, x_{t}\right) \approx \mathcal{R}_{e}\left[\frac{e^{-\alpha k_{u}}}{\pi} \sum_{j=1}^{N} e^{-i \frac{2 \pi}{N}(j-1)(u-1)} \mathrm{Y}\left(u_{j}, y_{t}, j, x_{t}\right) e^{i b u_{j}} \frac{\eta}{3}\left[3+(-1)^{j}-\delta_{j}\right]\right]
$$

The presented condition generates a tradeoff between precision of the integral approximation and step size of the log strike partitions. However the fractional Fourier transform (FRFT) allows to use independent log strikes step size and integration grid precision Chourdakis (2004). Under the risk neutral measure, we consider a couple of semi Markov spot price log-jump densities $b_{i, j}$, when the market switches from regime $i$ to $j$, and we denote them $\kappa_{i, j}$ with: 


$$
\kappa_{i, j}=\left\{\begin{array}{l}
1-\varepsilon_{i, j} \text { with probability } p_{i, j} \\
1 \text { with probability } 1-2 p_{i, j} \\
1+\varepsilon_{i, j} \text { with probability } p_{i, j}
\end{array} \text { with } \varepsilon_{i, j}>0, p_{i, j} \in\left[0, \frac{1}{2}\right], \forall i, j \in I(1, m) .\right.
$$

with

$$
\varepsilon_{i, j}>0, p_{i, j} \in[0,], \forall i, j \in I(1, m) .
$$

We consider the following partition $0=a_{0}<a_{1}<\ldots<a_{M-1}=T^{\star}$ of $\left[0, T^{\star}\right]$ and for convenience we denote $a_{M}=\infty$. We assume that the conditional intensity of the semi Markov process $\theta_{t}$ is a piecewise constant approximation of Weibull intensities. This is guided by a couple of motivations: first its flexibility and then the necessity of Lie bracket condition (30). On the one hand, Weibull intensities are quite flexible as they can simulate increasing, constant and decreasing rates. On the other hand, the Lie bracket condition is satisfied piecewise, since the $\lambda_{i, j}$ are piecewise constant. The piecewise conditional intensity approximation of Weibull intensities relative to the partition $(a)_{k=0}^{M-1}$ are defined:

$$
\begin{aligned}
\lambda_{i, j}\left(y_{s}\right) & =\left\{\begin{array}{l}
p_{i, j} \sum_{k=0}^{M-1} \frac{\vartheta_{i}}{S_{i}}\left(\frac{a_{k}^{\star}}{S_{i}}\right)^{\vartheta_{i}-1} 1_{\left[a_{k}, a_{k+1}\right)}\left(y_{s}\right) \text { if } i \neq j \\
-\sum_{j=1, j \neq i}^{m} \lambda_{i, j}\left(y_{s}\right) \text { otherwise }
\end{array}\right. \\
& =\left\{\begin{array}{l}
\alpha_{i, j} \sum_{k=0}^{M-1}\left(a_{k}^{\star}\right)^{\gamma_{j}-1} 1_{\left[a_{k}, a_{k+1}\right)}\left(y_{s}\right) \text { if } i \neq j \\
-\sum_{j=1, j \neq i}^{m} \lambda_{i, j}\left(y_{s}\right) \text { otherwise, }
\end{array}\right.
\end{aligned}
$$

$\forall s \in[0, T]$, with $\alpha_{i, j}=p_{i, j} \frac{\vartheta_{i}}{S_{i}^{\vartheta_{i}}}$ and $a_{k}{ }^{\star}=\frac{a_{k}+a_{k+1}}{2}, \forall k \in I(0, M-2)$. Three notable sets of parameters are absent from most option price formulas whenever the market is subjected to Markov regime changes: the backward recurrence time, the semi Markov sojourn time distribution, and the price jumps associated with regime changes, respectively. We will examine the added flexibility of stock price models under semi Markov regimes due to the extra parameters and the impact of each of the first two parameters on option prices and implied volatilities. We first make a couple of observations necessary to shed more light on the simulation results. If the intensity function is a continuous function of the backward recurrence time, from the derivative of the matrix exponential under the Lie bracket condition Magnus (1954), from (27) and (31) we have:

$$
\begin{aligned}
& \frac{\partial C}{\partial y}(t, T, k, y, j, x)=\frac{e^{-\alpha k}}{2 \pi} \int e^{-i u k} \frac{\partial \mathrm{Y}}{\partial y}(t, T, u, y, j, x) \\
& =\frac{e^{-\alpha k}}{2 \pi} \int_{u \in \llbracket}\langle[M(u-i(1+\eta), y+t)-M(u-i(1+\eta), y)] \\
& \left.\times \exp \left(\int_{y}^{t+y} M(u, \eta) d \eta\right) e_{j}, e^{i u(\ln (x)-k)} \mathbf{1}\right\rangle d u,
\end{aligned}
$$

where the difference of matrices in (46) is performed component-wise and yields,

$$
\begin{aligned}
& M_{p q}(u-i(1+\eta), y+t)-M_{p q}(u-i(1+\eta), y)
\end{aligned}
$$

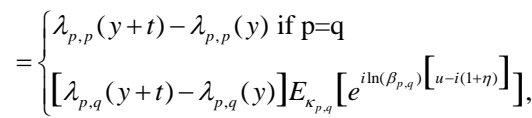


where $E_{\kappa_{p, q}}$ is the expected value with respect to $k_{p, q}$. (47) shows an interesting feature of option prices in semi Markov market regimes. If the rate matrix $\left(\lambda_{i, j}(y)\right)_{m \times m}$ is monotonic component-wise, the backward recurrence time effect on option prices is most significant for long range maturity options. Assuming the intensity functions $\lambda_{i, j}$ have finite limits when the backward recurrence time grows to infinity, we have

$$
\lim _{y \rightarrow \infty} \frac{\partial C}{\partial y}(t, T, k, y, x)=0, \forall i, j \in E .
$$

Hence, the semi Markov conditional intensity matrix is asymptotically constant, which implies that asymptotically, semi Markov market regime prices are identical to Markov market regime option prices. Examining Figures 6, 7 and 8 shows that irrespective of the model, option prices from prices processes in Markov market regimes can be sandwiched between semi Markov market regimes with shape parameter $\vartheta$ above and below 1. More importantly, the specific observation that prices from semi Markov market regimes with $\vartheta_{i}<1$ and $\vartheta_{i}>1$ are higher and lower than Markov prices, respectively is consistent with the underlying mathematical and economic theory. Indeed, our price model accounts for two sources of risk, the Levy and the semi Markov switching risks. The former is hedged against by the conditional minimum entropy martingale measure as the Levy jump process is turned into a martingale, and the latter isn't considered hedgeable and directly affects option prices. Hence, the higher the regime switching risk the larger the option price. From (45), choosing $\vartheta_{i}<1$ (resp. $\vartheta_{i}>1$ ) implies a decreasing (resp. increasing) transition rate, which translates in a decreasing (resp. increasing) regime switching risk. Simulations for Figures 6 and 7 are performed with $y$ $=.1$ year and $\vartheta_{i}<1$, hence, $\lambda_{i, j}(y)$ is largest for $\vartheta_{i}<1$, which justifies why prices for $\vartheta_{i}<1$ lie above prices corresponding to $\vartheta_{i}=1$ and $\vartheta_{i}>1$,respectively. Simulation of Figure 8 was performed with $y=1.1$ year. It shows that option prices are higher for larger shape parameters. This is in agreement with (45) as it shows that $\lambda$ is higher for higher values of $\vartheta_{i}$. One of the most documented shortcomings of Levy models for price processes is their inability to capture long term implied volatility smiles, Mitra (2009). Markov switching Levy price models succeed in slowing the dampening of the implied volatility smiles through the conditional intensity rate matrix, Mitra (2009). In our context, the conditional rate matrix is time dependent and could be affected by $y, \alpha$ and $\vartheta$, hence offering more control than Markov market regime models over long term smiles. Figure 15 shows that, irrespective of the Levy process used, long term smiles an smirks which often vanish at $T=1$ year in Markov regimes Bollen (1998), are still persistent at $T=2$ years when market regimes are semi Markovian. In addition, Figures 16, 17 and 18 show that the backward recurrence time, the shape and the scale parameters do have a prominent effect on the implied volatility surface. The three rows of Figure 9 display three different effects of the backward recurrence time on the difference in prices between all market regimes, based on three different values of the shape parameter $\vartheta$. The first and the third row were simulated based upon $\vartheta_{i}\left\langle 1, \forall i \in E\right.$ and $\vartheta_{i}>1, \forall i \in E$, respectively. Option prices are decreasing in the first row and increasing in the third row in all market regimes. However, in each regime, prices decrease or increase at different rates, hence affecting the price differences between market regime prices. One therefore observe either an exacerbation or a reduction of differences in regimes as evidenced in the first and third row. The second row corresponds to $\vartheta_{i}=1, \forall i \in E$ and shows no change in option prices as the price model reduces to Markov market regime price model which is independent of $y$. Indeed, from (45), when $\vartheta_{i}=1, \forall i \in E, \lambda_{i, j}$ will reduce to a mere constant and will therefore be free of $y$. Similar observations are made in Figures 10 and 11. One also note from Figure 12, 13 and 14 that $y, \alpha$ and $\vartheta$, respectively, affect in-the money, at-the-money and out-of-the-money options prices and leave deep in-the-money (low call strikes relative to the spot price)and out-of-the-money (high call strikes relative to the spot price) option prices relatively unchanged. This stems from (26), where the payoff vanishes or grows substantially causing the option price to vanish or grow as well irrespective of the market regime when the log strike $k$ grows or decreases relative to the spot price respectively.

\subsection{FST Pricing of Vanilla and Exotic Option Contracts}

We first consider the pricing of two vanilla option contracts: single asset European option contracts and single asset American option contracts. We recall that American option contracts can be exercised any time before expiration of the contract, unlike European option contracts which are settled at maturity. It has been shown Elton et al. (2009) that it is not optimal to exercise a non-dividend-paying American option contract before maturity. Hence, American and European call options contracts have the same price provided that the underlying asset does not pay dividends, Grabbe (1983). One will therefore be concerned only with pricing and comparing prices induced by Carr \& Madan (1999), and the FST numerical methods, R Jackson (2009), respectively. The FST pricing of European option contracts requires one time-step despite the assumption of time dependent conditional intensity matrix. It is based on (40) applied at $(t, y, x)$ as follows: 


$$
\mathbf{C}(t, T, K, y, x)=\mathcal{F}^{-1}\left[\exp \left[\int_{y}^{T-t+y} M(w, s) d s\right] . \hat{S}(w, K) . \mathbf{1}\right]
$$

Simulation parameters used for pricing purpose are as follows: $m=3$ market states, interest rate $r=.05$, spot price $S=$ $100, \sigma=(.3, .5, .7), \alpha=(-3,2,1 ; 2,-6,4 ; .5,1,-1.5), \vartheta=(5,3, .3), \epsilon=(0, .2, .1 ; .4,0, .1 ; .1, .3,0)$ and the jump and drop probabilities $p=(0, .2, .1 ; .25,0, .3 ; .1,0,0)$. We notice from Figure 4 that SMBS call option prices obtained from FST and Carr and Madan are identical up to the third decimal as is the case with call option in Markov regime markets Momeya (2012). However, the error plot shows that Carr and Madan prices are consistently slightly larger than FST prices. As for the pricing of American options in semi Markov markets, (40) allows us to use the FST method, R Jackson (2009), thus far applied to Markov regime markets. The FST is applied based on the discretization scheme presented in Remark 4.2. American put option prices are larger than their payoff, as they can be either exercised or held at each time step. The option holder always chooses the alternative netting the larger benefit. Such a maximum condition is enforced in the design of the pricing algorithm as follows:

$$
\mathbf{C}^{\star}\left(t_{n}, T, K, y, x_{n}\right)=\mathcal{F}^{-1}\left[\exp \left[\int_{y}^{y+\Delta t} M(w, s) d s\right] . \mathbf{C}\left(t_{n+1}, T, K, y, w\right)\right]
$$

which is the holding price of the option at time $t_{n}$, while the price of the option at the same time is

$$
\mathbf{C}\left(t_{n}, T, K, y, x_{n}\right)=\max \left(\mathbf{C}^{\star}\left(t_{n}, T, K, y, x_{n}\right), \mathbf{C}\left(T, T, K, y, x_{n}\right)\right),
$$

where the maximum is applied component wise.
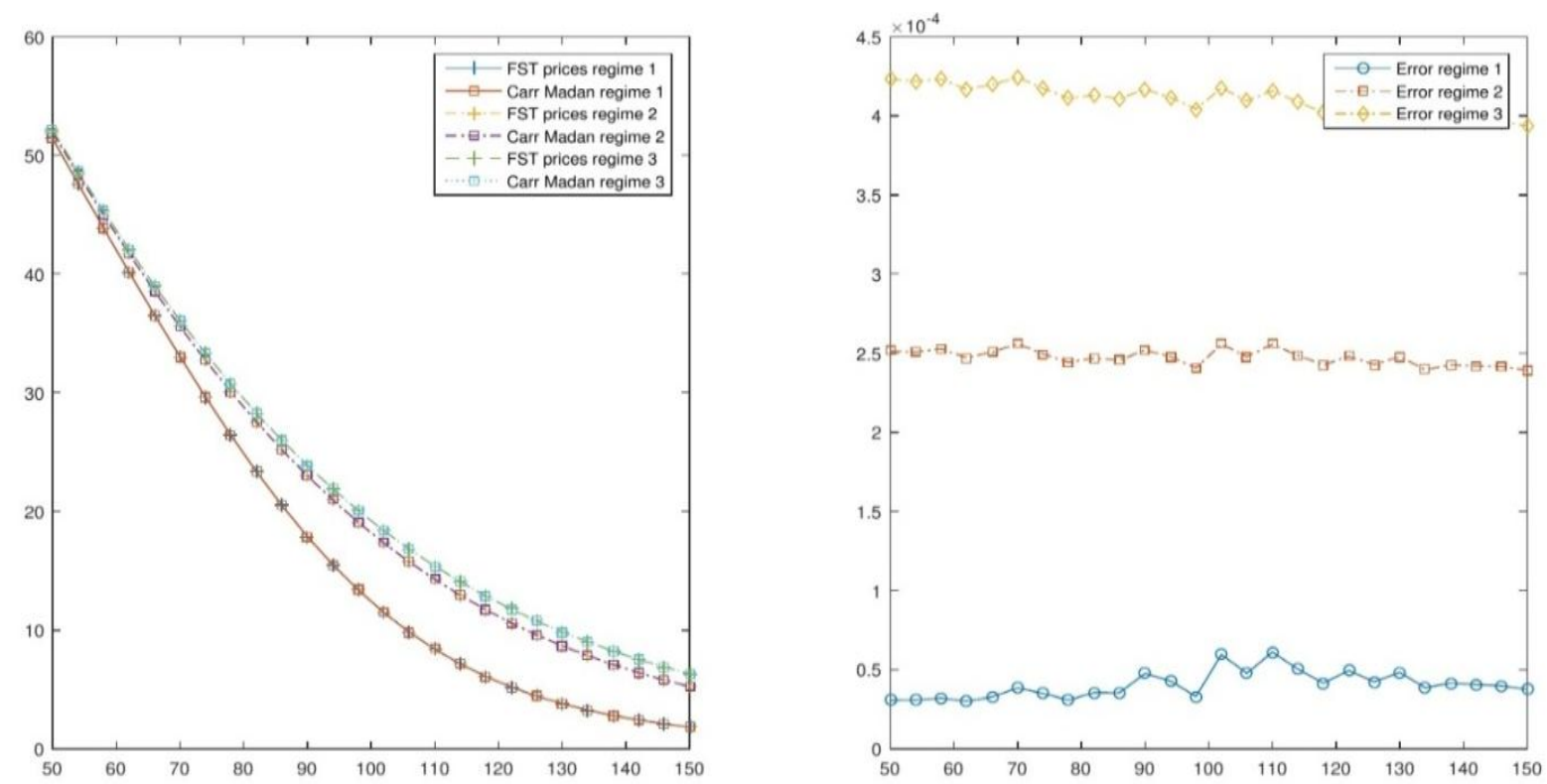

Figure 4. The left-hand side plot presents comparisons of Semi Markov Back Scholes FST and Carr and Madan prices of European option in every state of the market while the right-hand side plot exhibits error differences in each regime.

We simulate prices of European style Digital and asset-or-nothing exotic option prices. We recall that at expiry, digital call option contracts pay $\$ 1$, if the spot price is at least at large as the strike and nothing otherwise. Digital put options however, pay nothing if the spot price is larger than the strike and \$1 otherwise. Asset-or-nothing option contracts are essentially the same as digital option contracts with the only difference that they pay the asset price worth or nothing. Their respective payoff functions can be expressed as follows: 


$$
S\left(x_{T}, k\right)=\left\{\begin{array}{l}
1_{\left(x_{T} \geq K\right)} \text { for digital calls, } \\
1_{\left(x_{T}<K\right)} \text { for digital puts, } \\
x_{T} 1_{\left(x_{T} \geq K\right)} \text { for asset-or-nothing calls, } \\
x_{T} 1_{\left(x_{T}<K\right)} \text { for asset-or-nothing puts. }
\end{array}\right.
$$

The pricing of this style of path-independent exotic option contracts in semi Markov regime switching could be done using the FST method with one single time step. The first row of Figure shows that the effect of the backward recurrence time on European vanilla observed in the preceding section carries over to American and exotic option contracts.
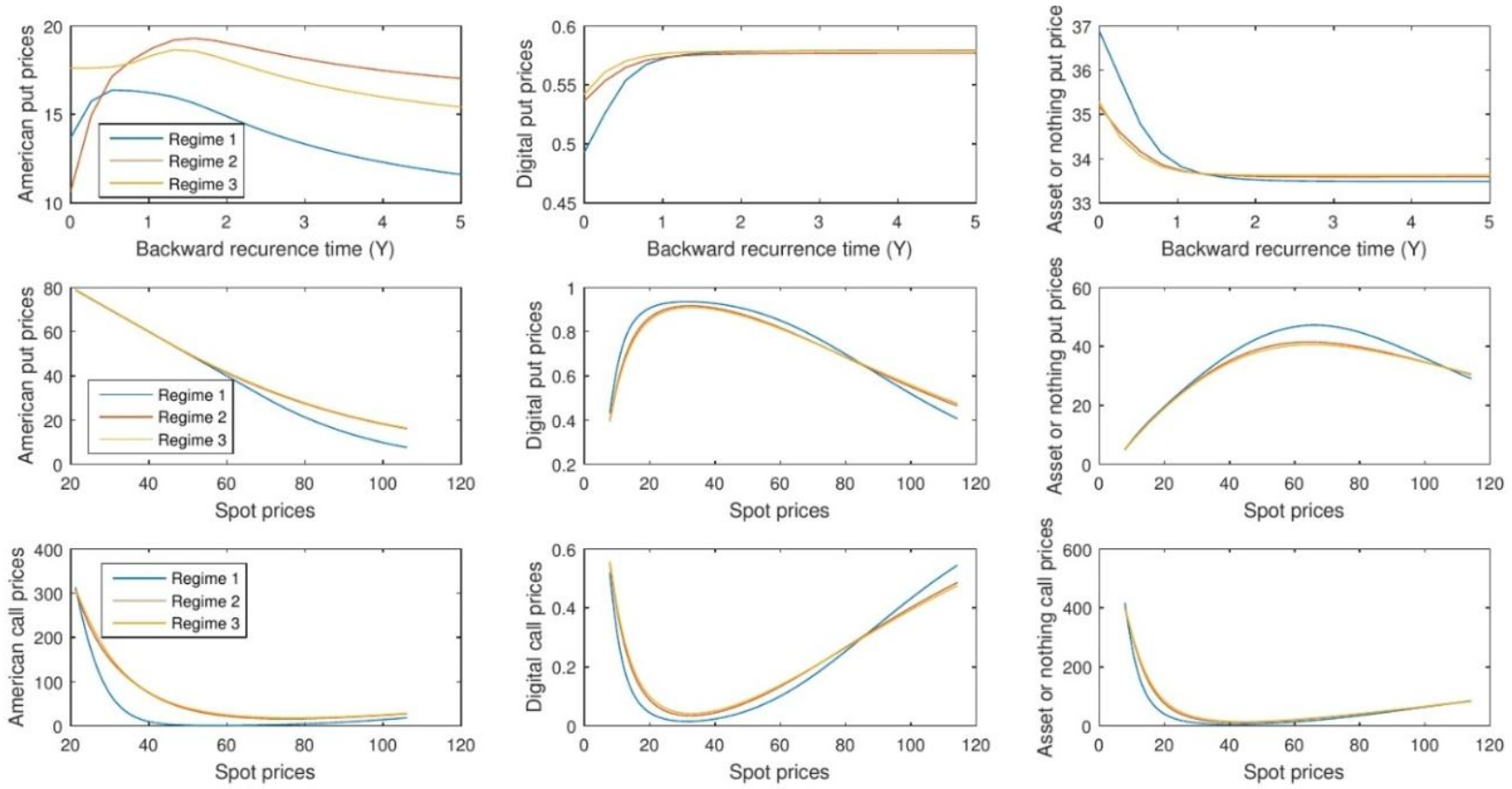

Figure 5. The First Row Captures the Effect of Backward Recurrence on American Style Put, Digital Put and asset-or-nothing Put Option Prices While the Second and Third Rows Present all Three Put and call Option Prices at each Market State. 

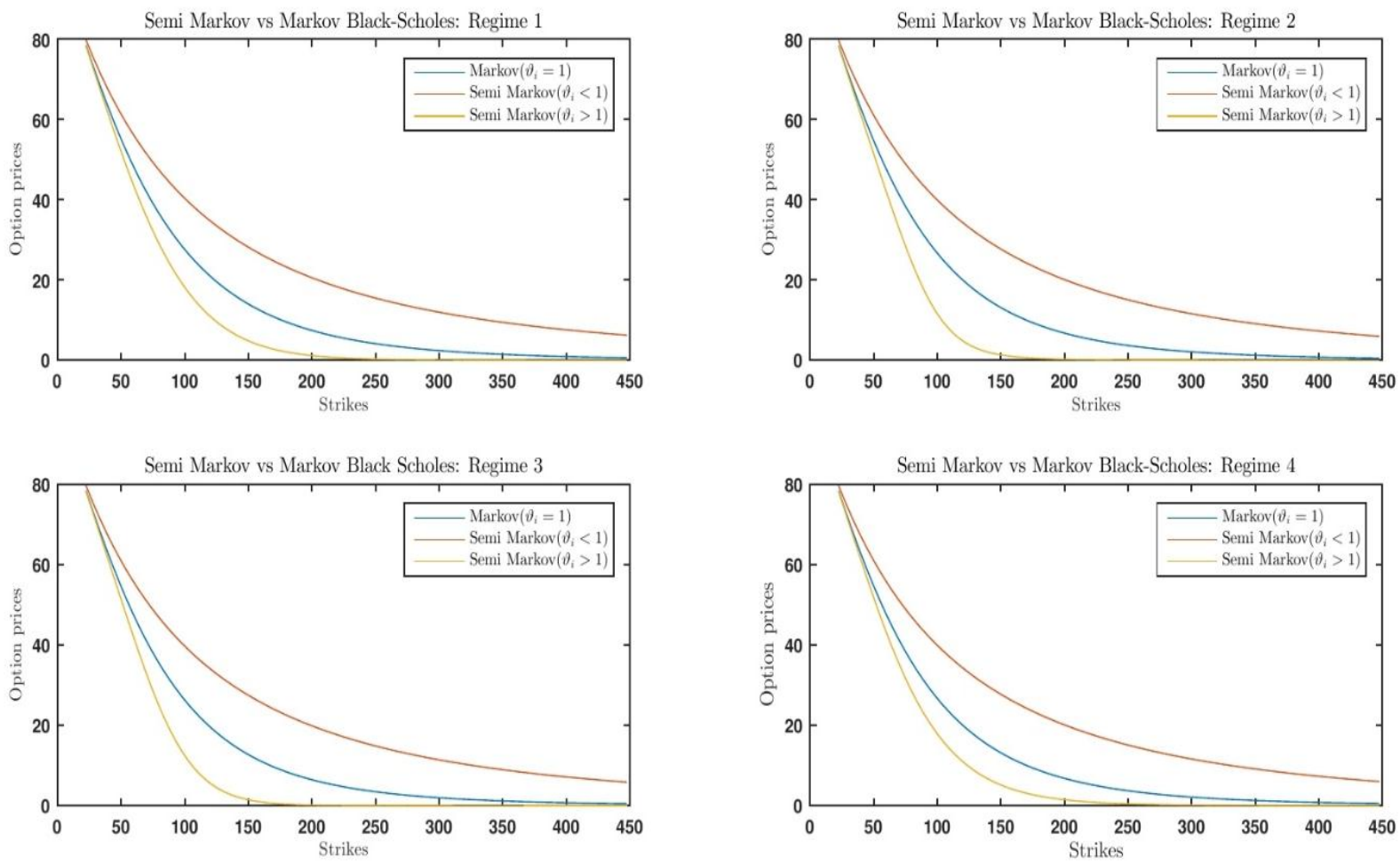

Figure 6. Comparison of Option Prices in a Markov Switching Black Scholes Model and a Semi Markov Switching Black Scholes (SMBS) Model.
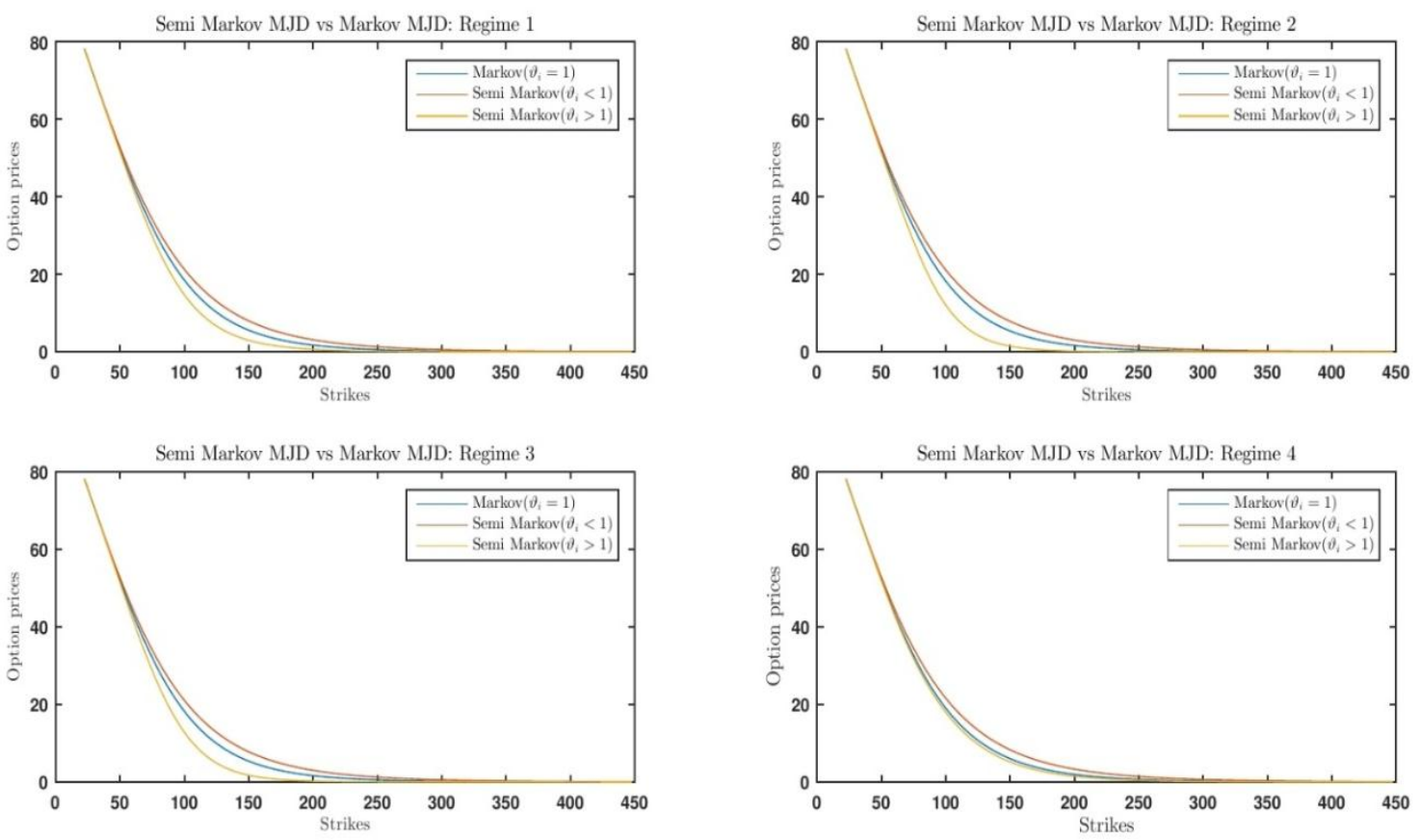

Figure 7. Comparison of Option Prices in a Markov Switching Merton Jump Diffusion Model and a Semi Markov Switching Merton Jump Diffusion (SMMJD) Model. 

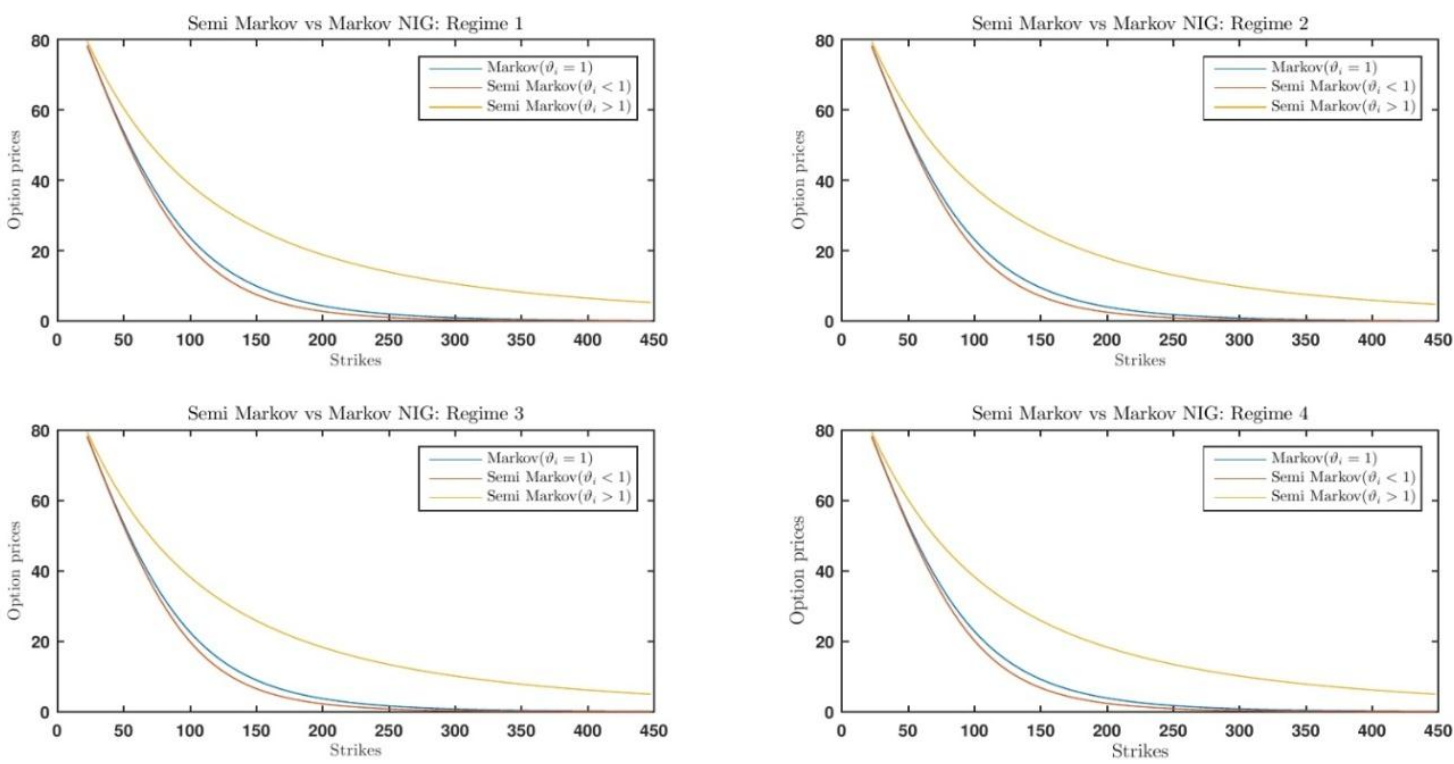

Figure 8. Comparison of Option Prices in a Markov Switching Normal Inverse Gamma Model and a Semi Markov Switching Normal Inverse Gamma (SMNIG) Model.
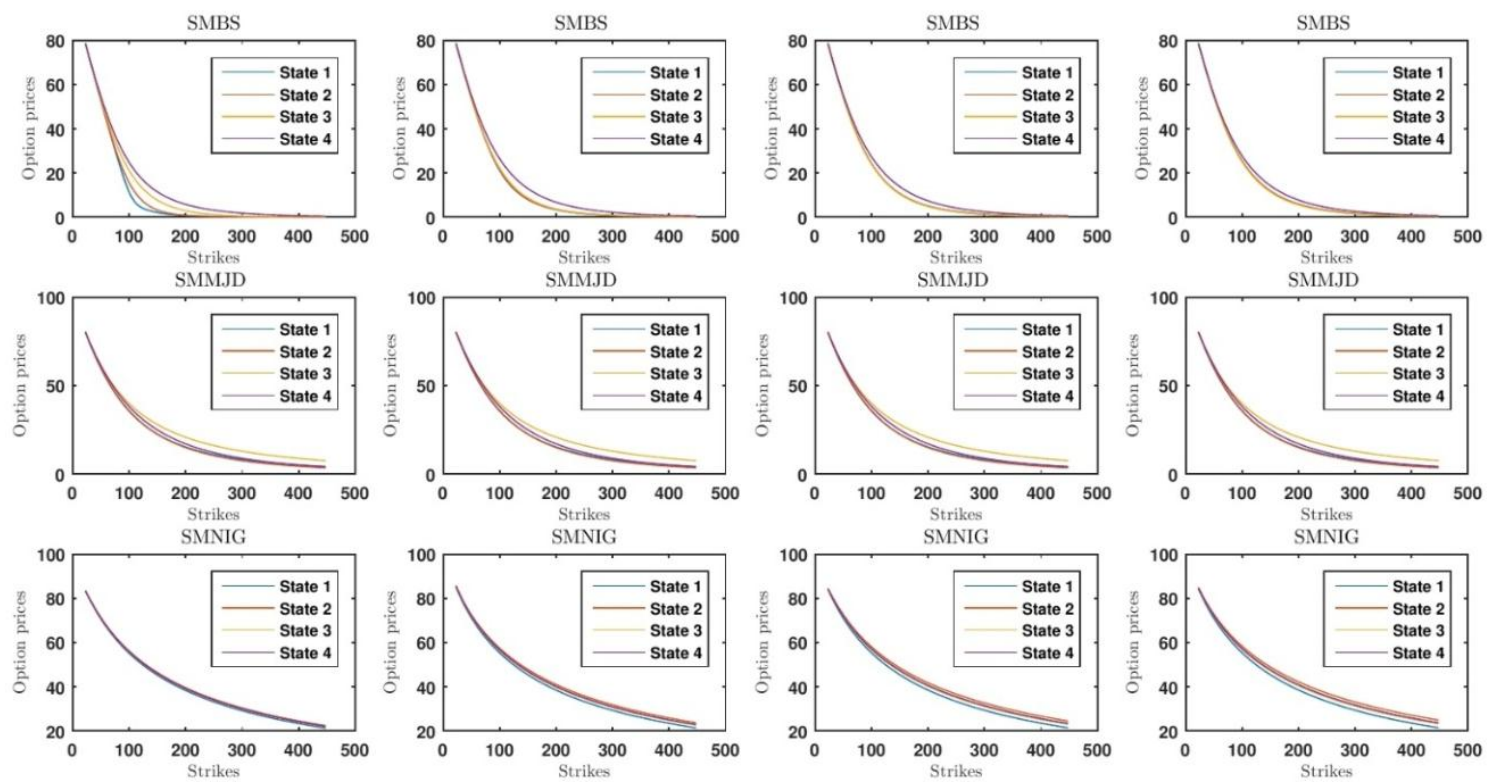

Figure 9. Effects of the Backward Recurrence Time on Option Price. The First, Second and Third Rows are Simulated with $\vartheta_{i, j}<1, \forall i, j \in E, \vartheta_{i, j}>1, \forall i, j \in E$ and $\vartheta_{i, j}=1, \forall i, j \in E$, respectively. 

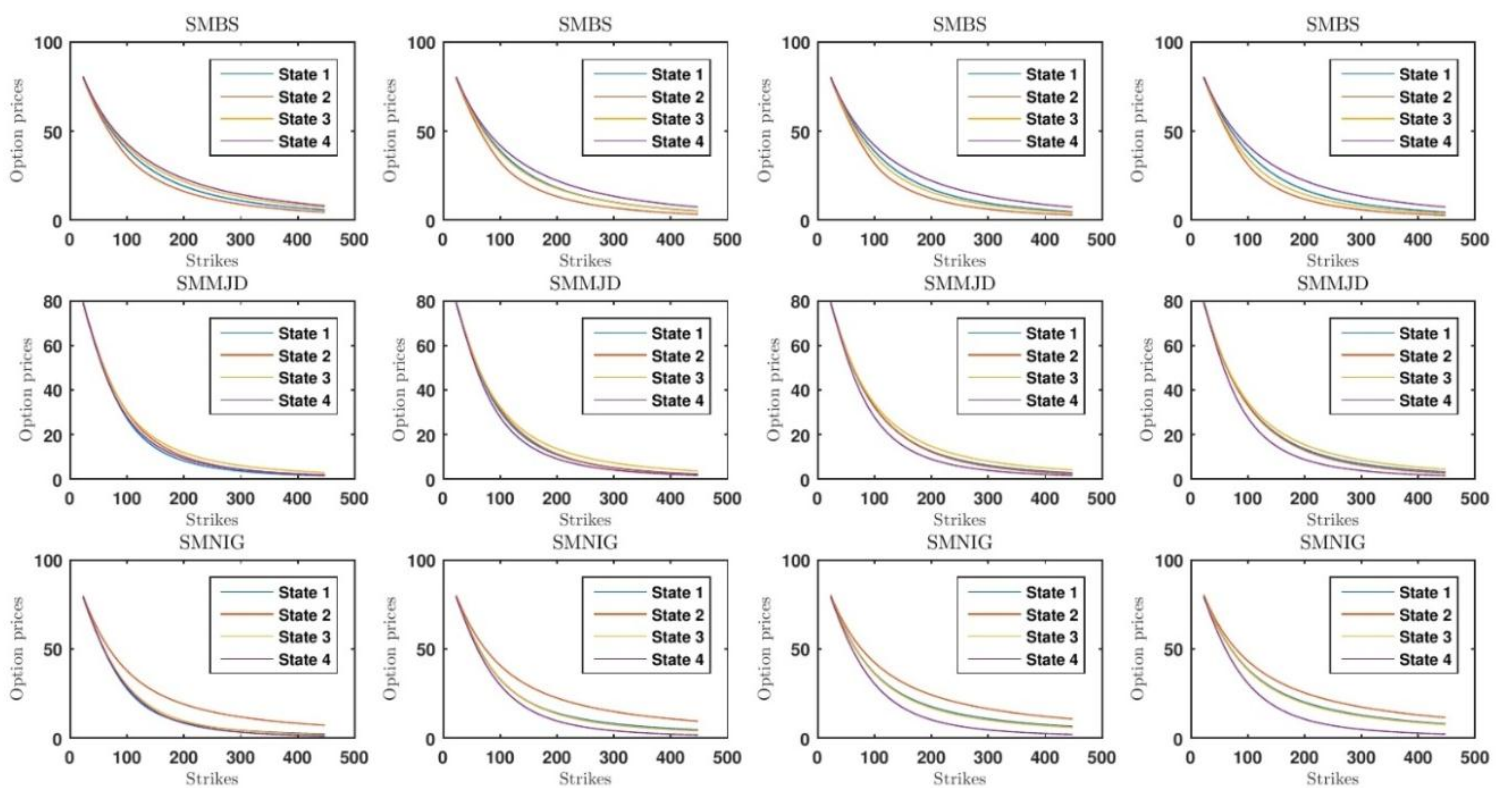

Figure 10. Effects of the Scale Parameter $\alpha$ on Option Prices $C$.
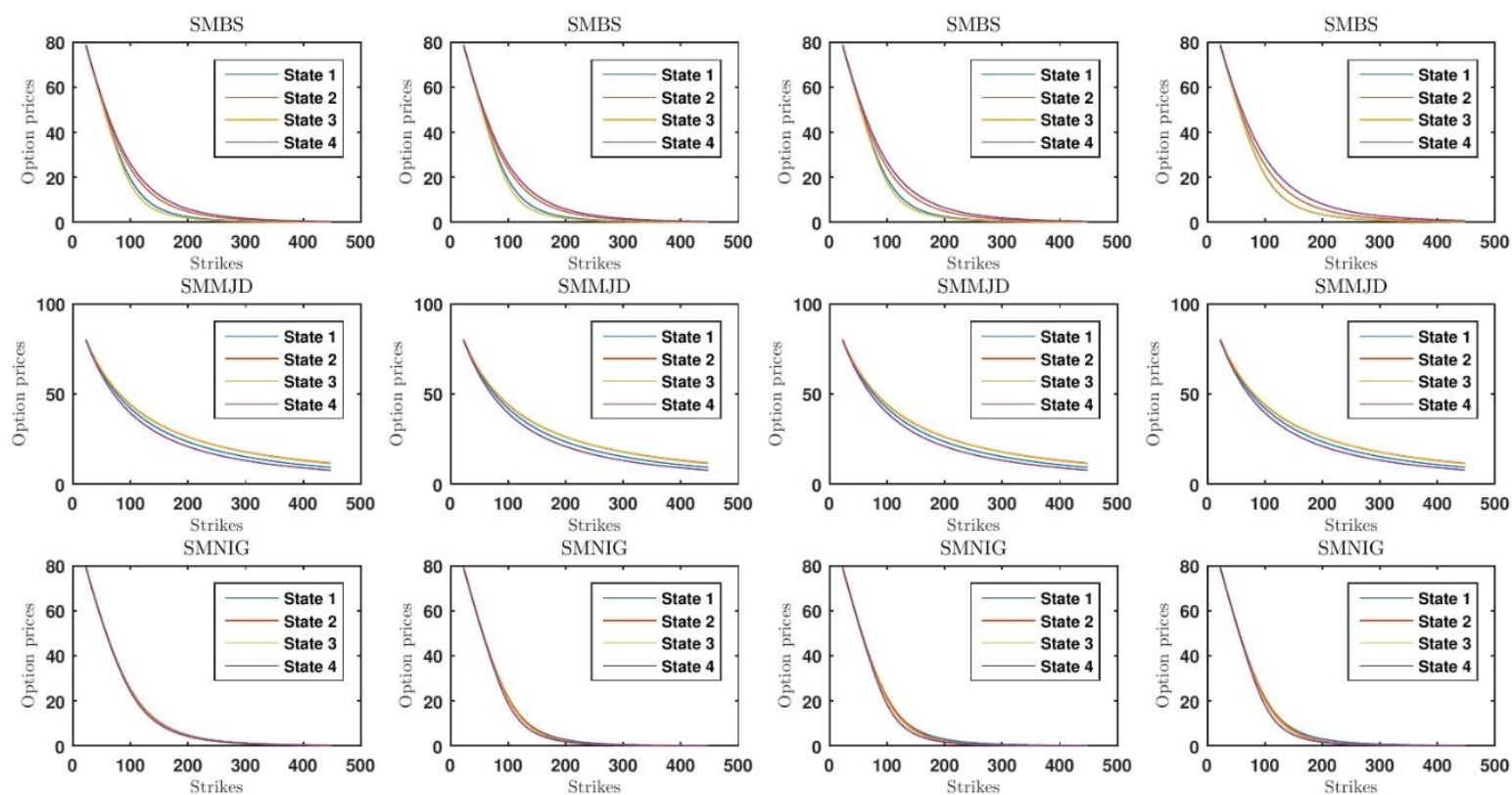

Figure 11. The Effects of the shape Parameter on option Prices. The shape Parameter Vector in the Simulation is $\zeta_{k} \vartheta$ where $\zeta_{k} \in\{.25, .5, .75,1\}$. 

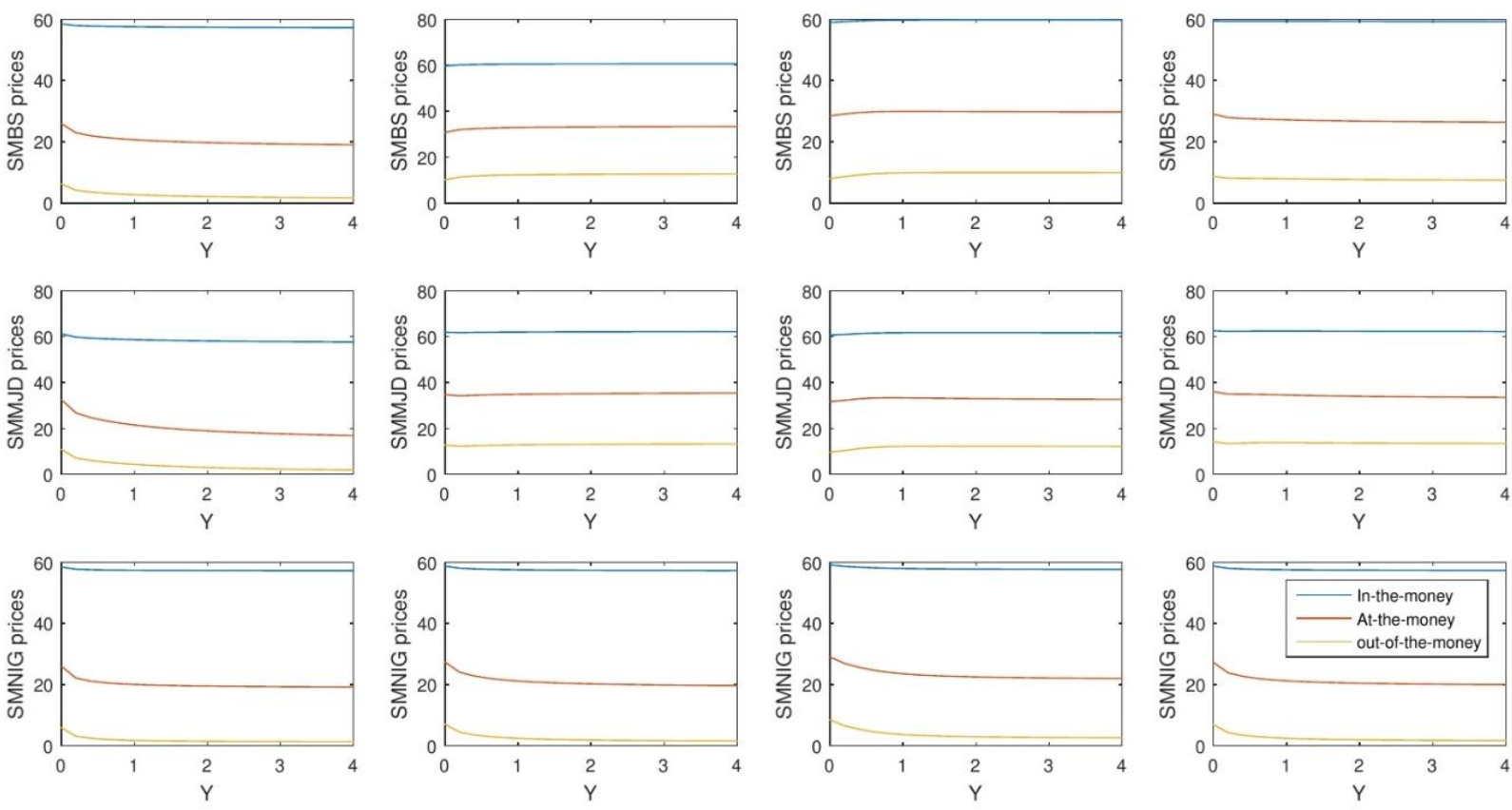

Figure 12. The Effects of the Backward Recurrence Time $y_{t}$ on option Prices $C$ from the Standpoint of the strike Price $K$ of the Option and the Model used (SMBS, SMMJD or SMNIG). Effects on Out of the Money Options (Orange), at the money (Red) and in the Money (Blue) are Compared.
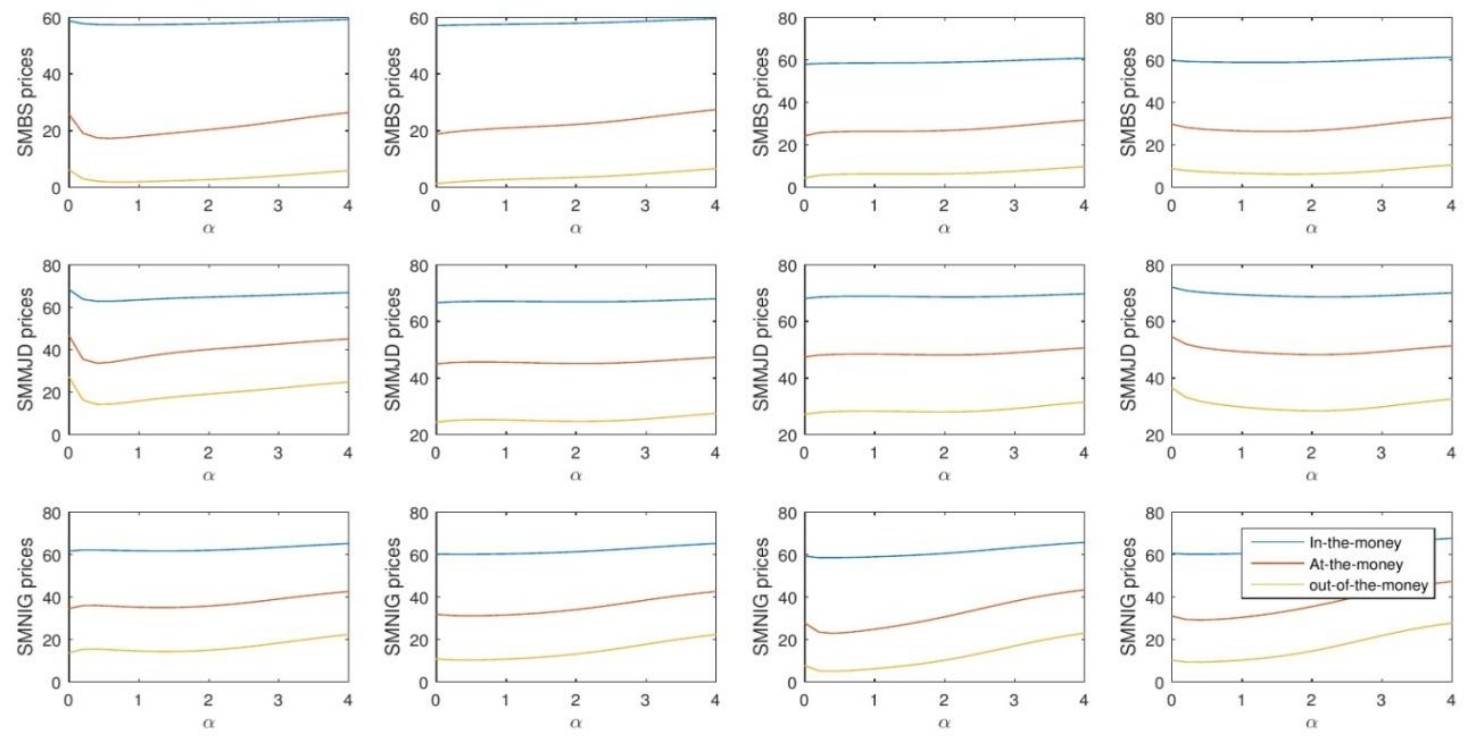

Figure 13. The Effects of the Scale Parameters $\alpha$ on Option Prices $C$ is Noticeable for All Three Models Regardless of the option'S Moneyness. Effects on Out of the Money Options (Orange), at the money (Red) and in the money Options

(Blue) are Compared. 

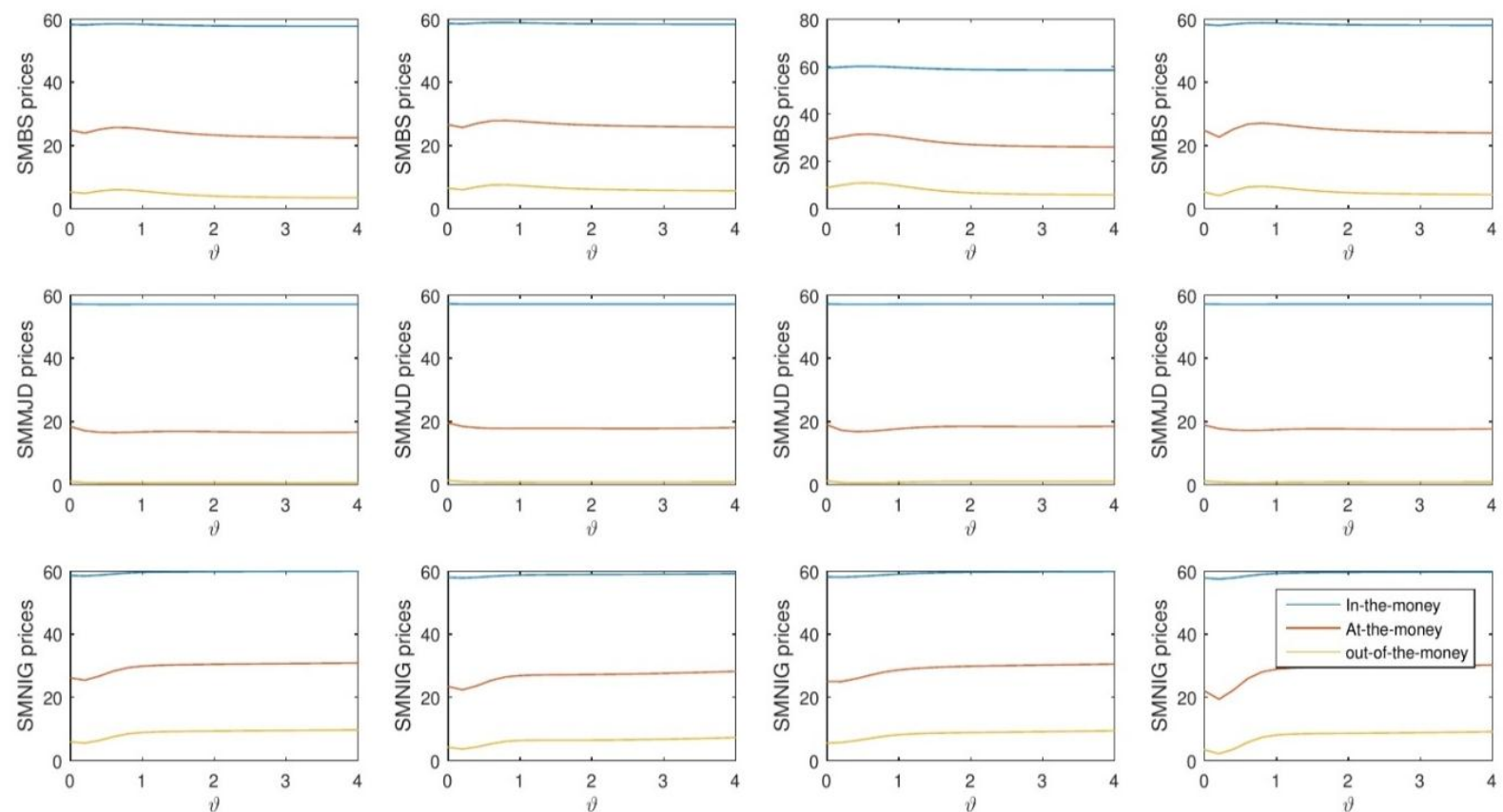

Figure 14. Effects of the Shape Parameters $\vartheta$ on Option Prices Care Noticeable for All Three Models and Regardless of the Option Moneyness. Effects on Out of the Money Options (Orange), at the money (Red) and in the money (Blue) are Compared.
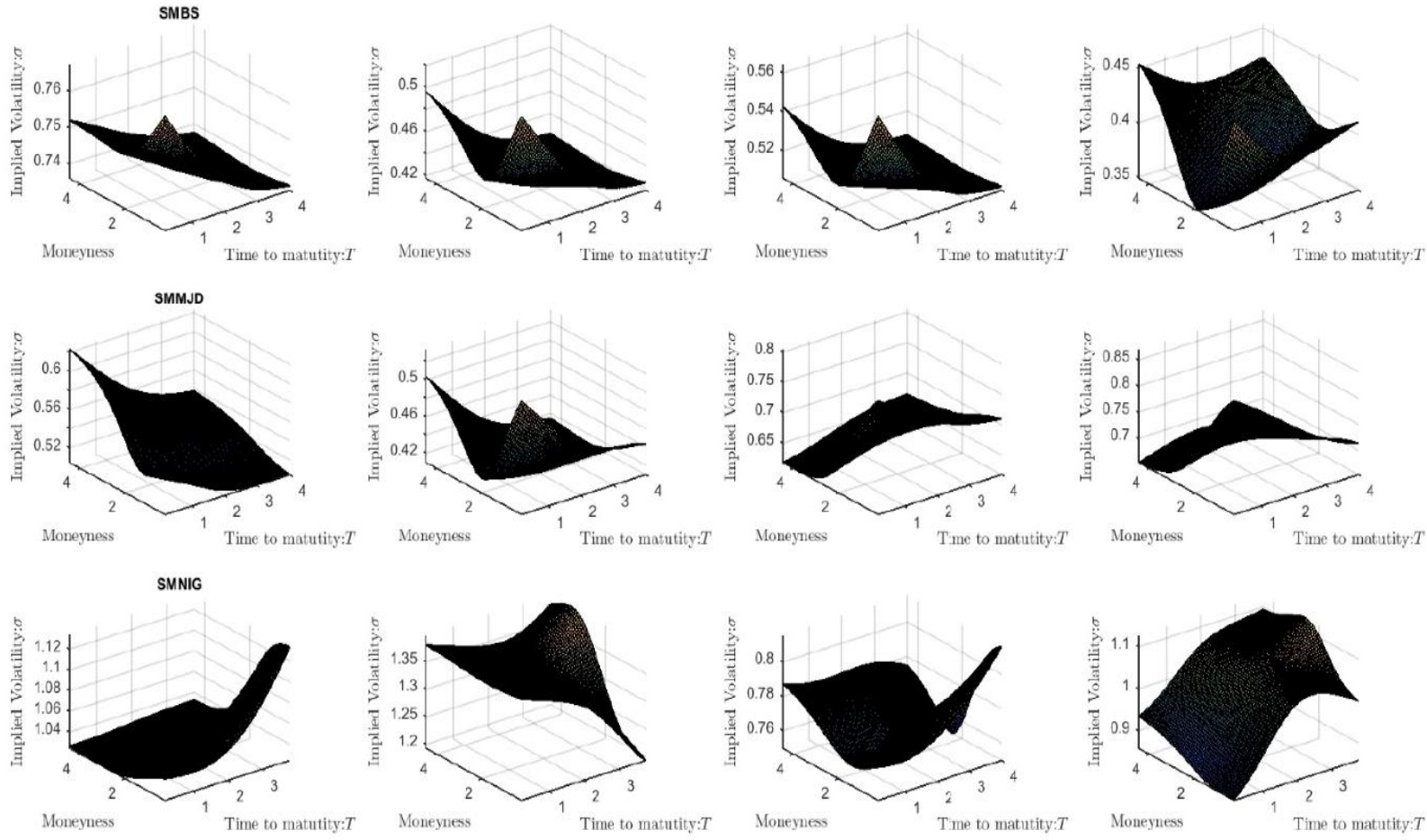

Figure 15. Implied Volatility Surfaces Induced by Option Prices Generated By SMBS (First Row), SMMJD

(Second Row) and SMNIG (Third Row). Column 1-4 Correspond to Market Regimes 1-4, Respectively. 


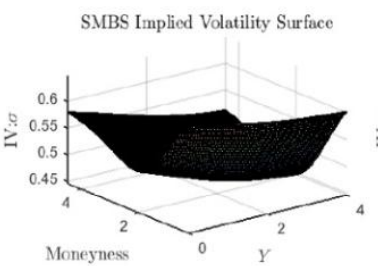

SMBS Implied Volatility Surface

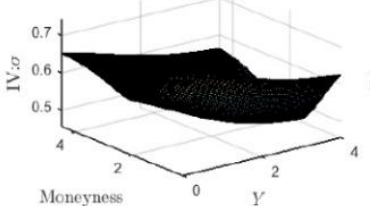

SMBS Implied Volatility Surface

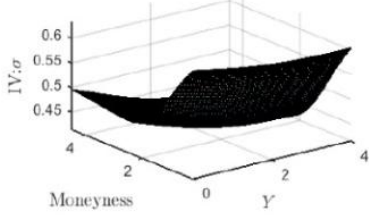

SMBS Implied Volatility Surface

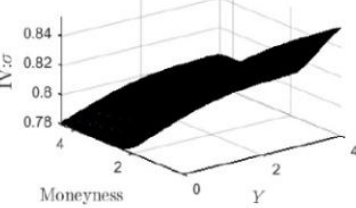

SMBS Impliod Volatility Surface

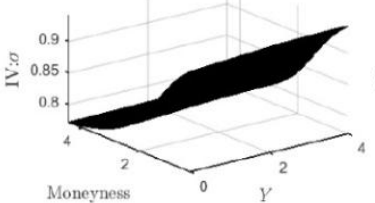

SMBS Implied Volatility Surface

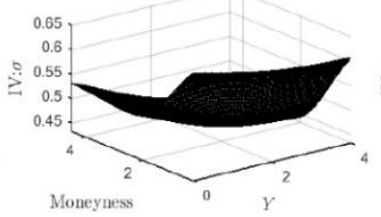

SMBS Implied Volat lity Surface

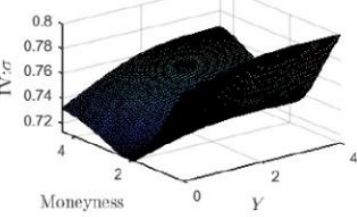

SMBS Impliod Volat lity Surfaco

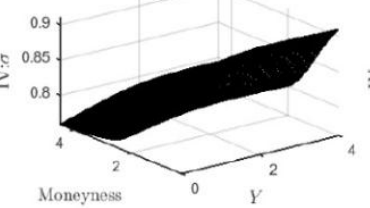

SMBS Implied Volat lity Surface

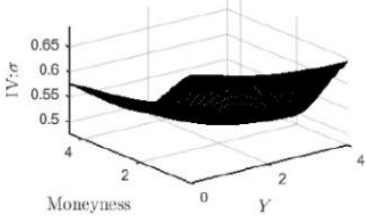

SMBS Implied Volatility Surface

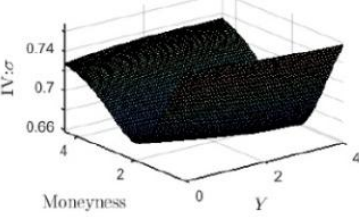

SMBS Impliod Volatility Surfaco

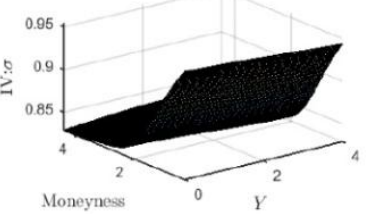

SMBS Implied Volatility Surface

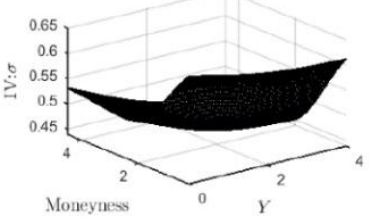

Figure 16. Effects of the Backward Recurrence Time $y_{t}$ on the Implied Volatility. The Models Simulated are as follows: SMBS (first row), SMMJD (second row) and SMNIG (third row). Column 1-4 correspond to Market Regimes 1-4, Respectively.
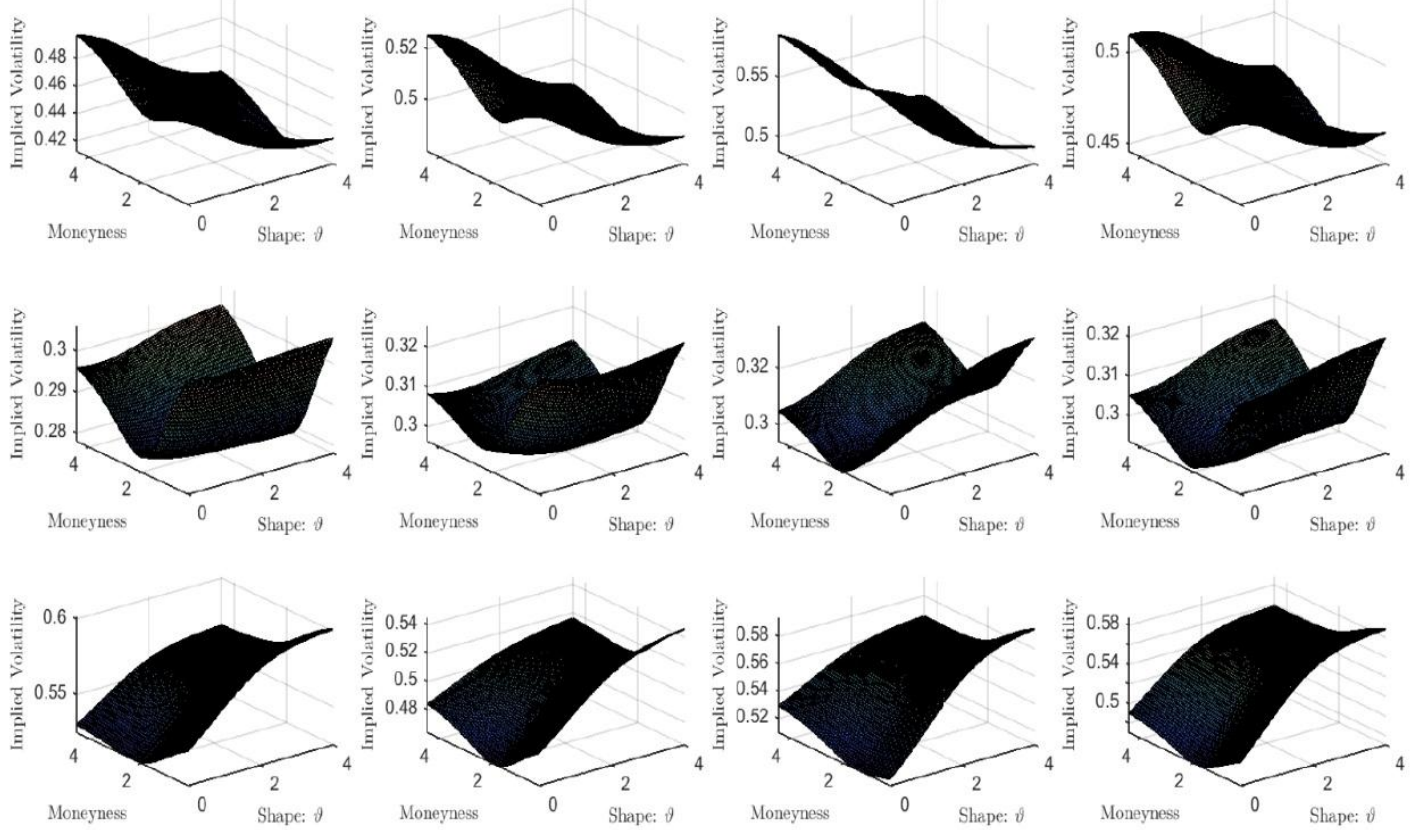

Figure 17. Effects of the Shape Parameter $\vartheta$ on the Implied Volatility. The models Simulated are as follows: SMBS (first row), SMMJD (second row) and SMNIG (third row). Column 1-4 correspond to Market Regimes 1-4, Respectively. 

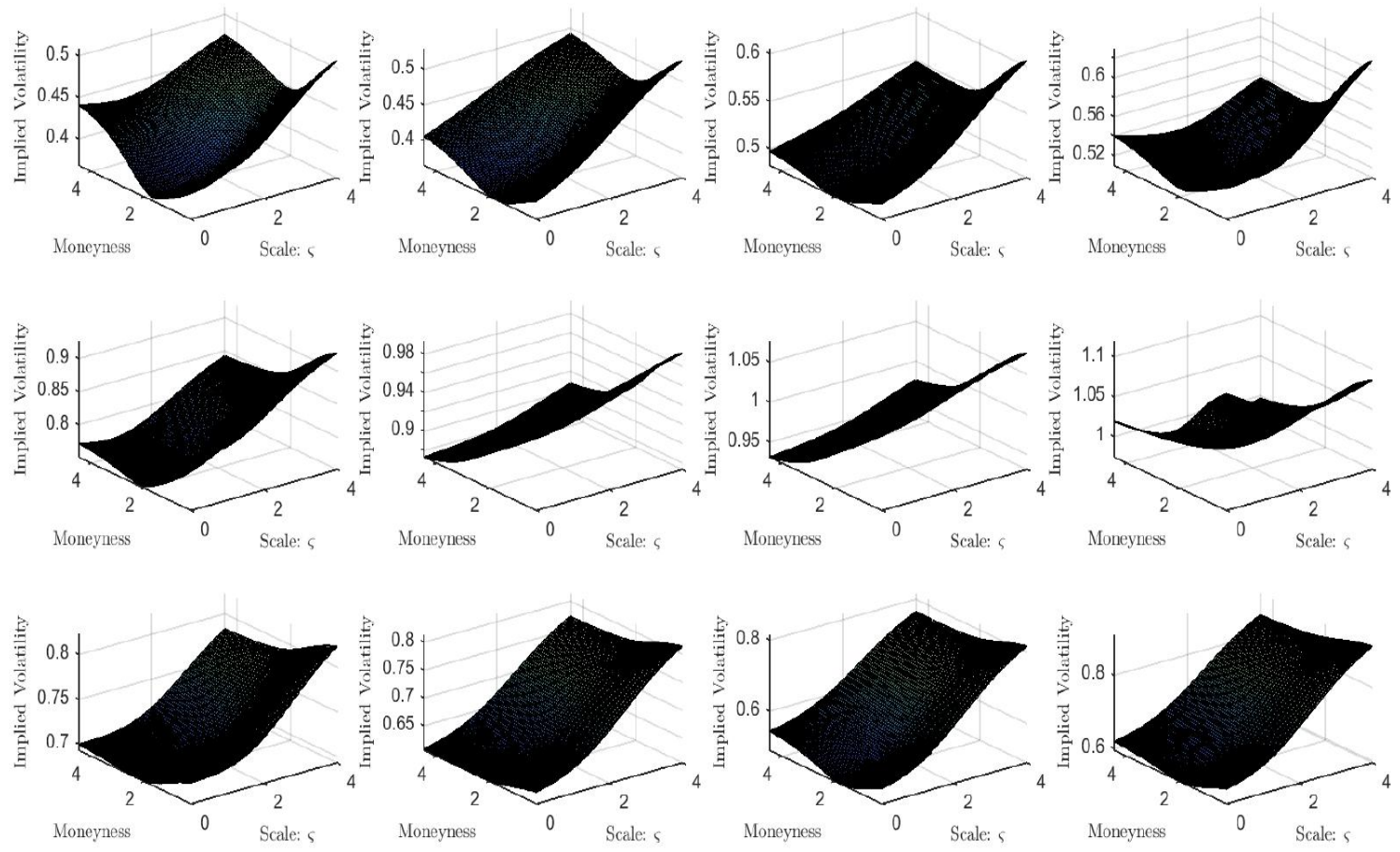

Figure 18. Effects of the Scale Parameter $\alpha$ on the Implied Volatility. The models Simulated are as follows:

SMBS (first row), SMMJD (second row) and SMNIG (third row). Column 1-4 correspond to market Regimes

$$
1-4 \text {, Respectively. }
$$

\subsection{Calibration}

In this section, we estimate the risk neutral parameters inducing option prices closest to observed market option prices in the least square sense. The Conditional Minimum Entropy Martingale Measure (CMEMM) is the risk neutral measure used for simulation purpose. Hence, the martingale condition (25) holds. The sojourn time distribution of the semi Markov process $\left(\theta_{s}\right)_{s \in[0, T]}$ is assumed to be piecewise exponential with intensity function defined in (45). The jump distribution at a regime switch is defined in (43) and (44). Option prices induced by Levy processes are well known to fit market prices better Tankov (2003), than BS induced option prices. It is therefore more appropriate to isolate the effects of market regime by calibrating regime switching BS parameters to market option prices. On the other hand, in Subsection 4.1, we have concluded that the effects of the backward recurrence and sojourn time distribution parameters increase as time to expiry goes up. Hence, our choice of the time to expiry $T=1.2$ years. We will use 4 data sets to calibrate semi Markov regime switching Black Scholes models. We will show that in our framework, calibration results provide a fit at least as good as Markov switching models with the added advantage of a more insight into the economic interpretation of market regimes. Data of interest are in-the-money and at-the-money European call option contract quotes on the Dow Jones Industrial Average Index (DJX) and the NASDAQ index(NDX), both collected March 2008 and March 2015, respectively. Data is presented in Table 3. We note that in-crisis DJX quotes of 2008 have been used in Deville (2007) to calibrate standard exponential Levy processes and in Momeya (2012) to calibrate Markov regime switching exponential Levy processes whereas post crisis data have been retrieved from the website www.optionseducation.org. The sum of squares (SS) and root mean square error (RMSE) are reported in Tables 4 and 5. Markov switching models are known to improve the fit of exponential Levy models discussed in Chourdakis (2005); Elliott \& Osakwe (2006); Naik (1993). It appears from Figures 19 and 20 that SMBS fits the market data at least as well as Markov switching BS and from 19 and 20 visibly better. Such a feature is hardly unexpected as the theoretical set up developed in Assonken \& G.S. (2015) and the corresponding estimation techniques parallel and extend the results of Chourdakis (2005); Elliott et al. (2005); Hainaut (2010); Momeya (2012); showing that Markov BS model estimation methods are nested inside SMBS. Furthermore, the parameter estimates of the sojourn time distribution of the semi Markov process shed an additional ray of light on the market regimes behavior. Although risk neutral parameters are a reflection of market-makers perception of the future, one can still glean a decent insight on the 
market behavior through calibrated parameters. One notices that calibrated 2008 DJX Markov regimes have two very similar regimes with nearly identically low volatilities (17.5\% and $17.6 \%)$, which reflects the market's widespread panic observed at the end of the year, hence suggesting that Markov regime models support a one state market. However, semi Markov parameters calibrated to the same data rather contend that $\alpha_{2}$ and $\vartheta_{2}$ are much higher than $\alpha_{1}$ and $\vartheta_{1}$, hence showing that the market will spend much more time in regime 2, the regime with the highest probability of price jumps or drops (regime $1,=.258$ ), which is in line with the sell-off observed throughout 2008 when the Dow Jones Industrial Average dropped by nearly $20 \%$ from June 2007 to June 2008. Post crisis Markov and semi Markov market state parameter estimates in Table 4 present the same conundrum as in-crisis parameter estimates. Indeed, Markov market model parameters describe a market with volatility non-reflective of the easing of the mood observed in the market. In fact, Markov market regime model supports evidence that volatility is higher in the post crisis market and the regime risks are similar. Another lingering effect of the financial crisis that has not been captured by Markov regime models is the remnant and even mounting fear of a market crash or correction which became even more acute since the DJIA and NASDAQ have reached all-time intraday highs May 192015 and April 23 2015, respectively. Both market features are captured by semi Markov parameters which provide a more intuitive interpretation of future behavior of the market regimes and crash fears. In fact, a look at the last line of Table 4 shows that volatility has decreased $(.143<.173$ and $.044<.141)$ while most of the remaining fear in the market is centered around unexpected crashes $\left(\square \square_{1}=18 \%\right.$, 1 $=.477)$. When the market is in state 1 seldom does it switch to state 2 as $\vartheta_{1}<\vartheta_{2}$ and $\alpha_{1}<\alpha_{2}$. However, the switching rate from state 2 to state 1 grows as $\vartheta_{2}$ is bigger than 1 . Hence, the market is expected to have short stays in state 2 which has low volatility $(.044<.144)$ and low probability of drop or jump $(.028<.477)$ and longer stays in the first state. This is also in line with the notion that the 2008 financial crisis has lingering effects and market makers expect significant market corrections and are incline to overreacting to new information. Similar observations are made from Table 5.

Table 2. European Call Option Quotes Written on the DJIA and NASDAQ during and after the financial crisis. Deep out-of-the-money options have been weeded out as they are of value close to zero. The spot prices are as follows: Dow Jones Industrial average, \$122 and \$180 for the 2008 and the 2015 data sets. Nasdaq Index \$1775 and \$4323

\begin{tabular}{|c|c|c|c|c|c|c|c|}
\hline \multicolumn{2}{|c|}{2008 DJX quotes } & \multicolumn{2}{|c|}{2015 DJX quotes } & \multicolumn{2}{|c|}{2008 NDX quotes } & \multicolumn{2}{|c|}{2015 NDX quotes } \\
\hline Strikes & Option prices & Strikes & Option prices & Strikes & Option prices & Strikes & Option prices \\
\hline 98 & 24.43 & 50 & 129.85 & 1400 & 334.95 & 4050 & 339 \\
\hline 99 & 23.40 & 55 & 124.85 & 1425 & 311.55 & 4075 & 319.1 \\
\hline 100 & 22.50 & 60 & 119.875 & 1450 & 288.35 & 4100 & 299.35 \\
\hline 101 & 21.55 & 65 & 114.9 & 1475 & 265.5 & 4125 & 280.15 \\
\hline 102 & 20.63 & 70 & 109.9 & 1500 & 242.6 & 4150 & 261.3 \\
\hline 103 & 19.68 & 75 & 104.925 & 1525 & 220.5 & 4175 & 242.95 \\
\hline 104 & 18.75 & 80 & 99.95 & 1550 & 198.95 & 4200 & 224.9 \\
\hline 105 & 17.83 & 85 & 94.975 & 1575 & 178.45 & 4210 & 217.9 \\
\hline 106 & 16.90 & 90 & 89.95 & 1600 & 158.55 & 4220 & 210.85 \\
\hline 107 & 15.98 & 95 & 85.05 & 1625 & 139.60 & 4225 & 207.4 \\
\hline 109 & 14.23 & 105 & 75.15 & 1675 & 104.45 & 4240 & 197.1 \\
\hline 110 & 13.33 & 110 & 70.225 & 1700 & 88.45 & 4250 & 190.4 \\
\hline 111 & 12.45 & 115 & 65.325 & 1725 & 73.8 & 4260 & 183.7 \\
\hline 112 & 11.63 & 120 & 60.425 & 1750 & 60.4 & 4270 & 177.7 \\
\hline 113 & 10.78 & 125 & 55.575 & 1775 & 48.45 & 4275 & 174.4 \\
\hline 114 & 9.95 & 130 & 50.75 & 1800 & 38.05 & 4280 & 171.2 \\
\hline 115 & 9.18 & 135 & 45.95 & 1825 & 29.2 & 4290 & 164.75 \\
\hline 116 & 8.40 & 140 & 41.25 & 1850 & 21.65 & 4300 & 158.6 \\
\hline 117 & 7.68 & 145 & 36.55 & 1875 & 15.65 & 4310 & 152.35 \\
\hline 118 & 6.93 & 150 & 32.025 & 1900 & 10.95 & 4320 & 146.25 \\
\hline 119 & 6.23 & 155 & 27.475 & 1925 & 7.45 & 4325 & 143.2 \\
\hline 120 & 5.58 & 160 & 23.125 & $\ldots$ & $\ldots$ & 4330 & 139.7 \\
\hline 121 & 4.95 & 165 & 18.925 & $\ldots$ & $\ldots$ & 4340 & 134.45 \\
\hline 122 & 4.35 & 170 & 15.1 & $\ldots$ & $\ldots$ & 4350 & 128.65 \\
\hline 124 & 3.25 & 180 & 8.075 & $\ldots$ & $\ldots$ & 4370 & 117.35 \\
\hline 125 & 2.74 & 185 & 5.275 & $\ldots$ & $\ldots$ & 4375 & 114.35 \\
\hline 126 & 2.28 & $\ldots$ & $\ldots$ & $\ldots$ & $\ldots$ & 4380 & 111.95 \\
\hline
\end{tabular}




$\begin{array}{cccccccc}127 & 1.90 & \ldots & \ldots & \ldots & \ldots & 4390 & 106.65 \\ 128 & 1.52 & \ldots & \ldots & \ldots & \ldots & 4400 & 101.1\end{array}$

Table 3. This Table reports calibration results of model parameters using option contracts on the Dow Jones Industrial Average(DJX). We assume the market has two regimes namely, $E=\{1,2\}$ and at inception of the contract, the market has been in its current state for $y=1.2 \mathrm{yrs}$. Only call options with maturity $T=47$ days are used for illustration.

\begin{tabular}{|c|c|c|c|c|c|c|c|c|c|c|c|c|c|}
\hline $\begin{array}{l}\text { Market } \\
\text { type }\end{array}$ & $\begin{array}{l}\text { Model } \\
\text { Type }\end{array}$ & $\alpha_{1}$ & $\alpha_{2}$ & $\vartheta_{1}$ & $\vartheta_{2}$ & $\varepsilon_{1}$ & $\varepsilon_{2}$ & $\hat{p}_{1}$ & $\hat{p}_{2}$ & $\hat{\sigma}_{1}$ & $\hat{\sigma}_{2}$ & SS & RMSE \\
\hline \multicolumn{14}{|l|}{ In-crisi } \\
\hline $\mathrm{s}$ & Markov & & & & & & & & & & & & \\
\hline \multirow[t]{2}{*}{ Market } & BS & 1.735 & 20.525 & 1 & 1 & 0 & 0 & 0 & 0 & .175 & .176 & 3.73 & .2454 \\
\hline & SMBS & .501 & 10.957 & 4.038 & 12.724 & .038 & .016 & .258 & .08 & .173 & .141 & 3.72 & .2449 \\
\hline \multicolumn{14}{|l|}{ Post } \\
\hline \multirow[t]{2}{*}{ Market } & BS & 3.54 & 20.025 & 1 & 1 & 0 & 0 & 0 & 0 & .25 & .224 & 24 & .65 \\
\hline & SMBS & 1.061 & 9.651 & 2.058 & 6.794 & .186 & .143 & .477 & .028 & .143 & .044 & 21 & .61 \\
\hline
\end{tabular}

Table 4.This Table Reports Calibration Results of Model Parameters using option Contracts on the NASDAQ (NDX). We Assume That The Market Has Two Regimes Namely, $E=\{1,2\}$ And At Inception Of The Contract, The Market Has Been In Its Current State for $y=1.2 y r s$. Only call Options with Maturity $T=47$ Days are used for Illustration.

\begin{tabular}{|c|c|c|c|c|c|c|c|c|c|c|c|c|c|}
\hline $\begin{array}{l}\text { Market } \\
\text { type }\end{array}$ & $\begin{array}{c}\text { Model } \\
\text { Type }\end{array}$ & $\alpha_{1}$ & $\alpha_{2}$ & $\vartheta_{1}$ & $\vartheta_{2}$ & $\varepsilon_{1}$ & $\varepsilon_{2}$ & $\hat{p}_{1}$ & $\hat{p}_{2}$ & $\hat{\sigma}_{1}$ & $\hat{\sigma}_{2}$ & SS & RMSE \\
\hline \multicolumn{14}{|l|}{ In-crisi } \\
\hline $\mathrm{s}$ & Marko & & & & & & & & & & & & \\
\hline \multirow[t]{2}{*}{ Market } & BS & 5.8 & 58.5 & 1 & 1 & 0 & 0 & 0 & 0 & .206 & .268 & 400 & 3.02 \\
\hline & SMBS & 14.402 & 18.389 & 24.306 & 7.166 & .012 & .017 & .096 & .102 & .003 & .196 & 384 & 2.95 \\
\hline \multicolumn{14}{|l|}{ Post } \\
\hline \multirow[t]{2}{*}{ Market } & BS & 11.68 & 44.493 & 1 & 1 & 0 & 0 & 0 & 0 & .172 & .159 & 993 & 4 \\
\hline & SMBS & 10.32 & 45.85 & 3.25 & 12.73 & .062 & .015 & .194 & .038 & .047 & .018 & 384 & 2.49 \\
\hline
\end{tabular}



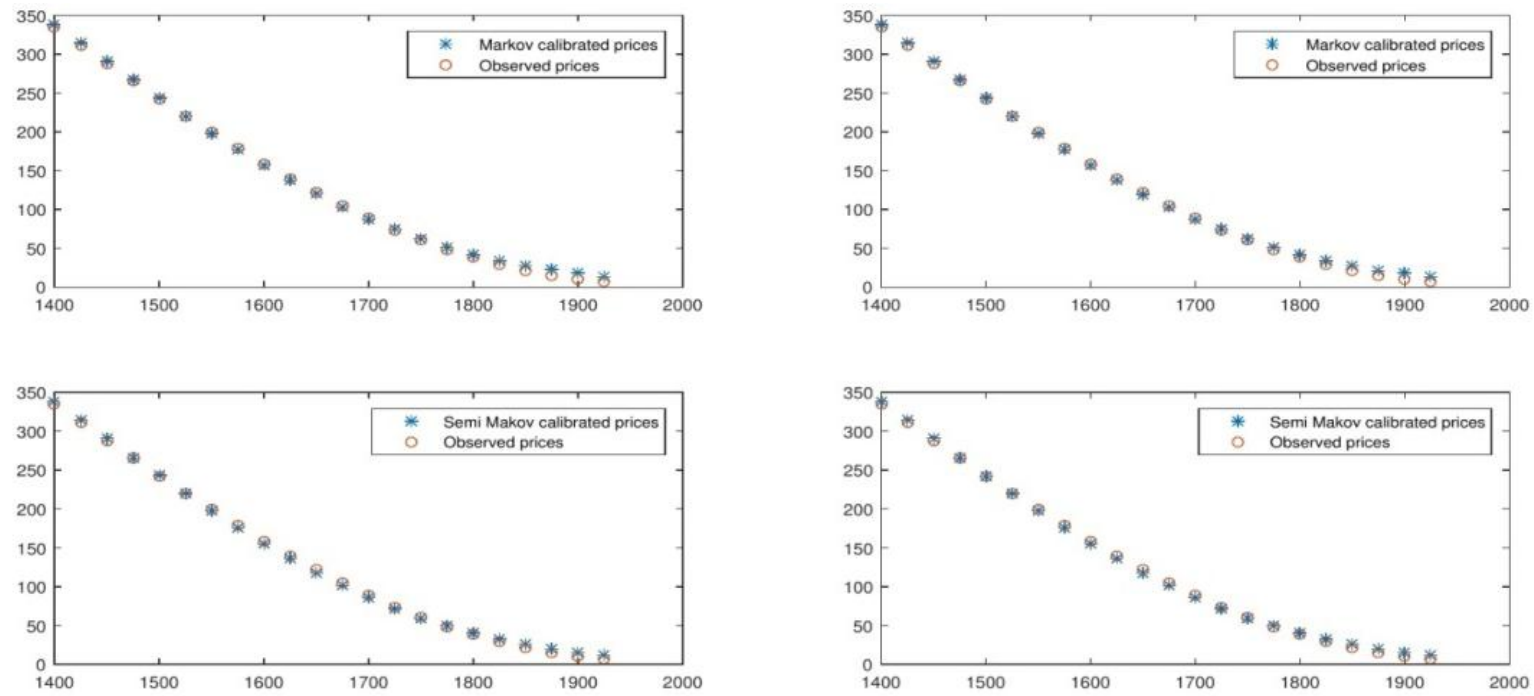

Figure 19.Calibration Results of the Markov and Semi Markov Regime Switching Models to NDX Observed Prices in 2008 with the Financial Crisis in Full Swing.
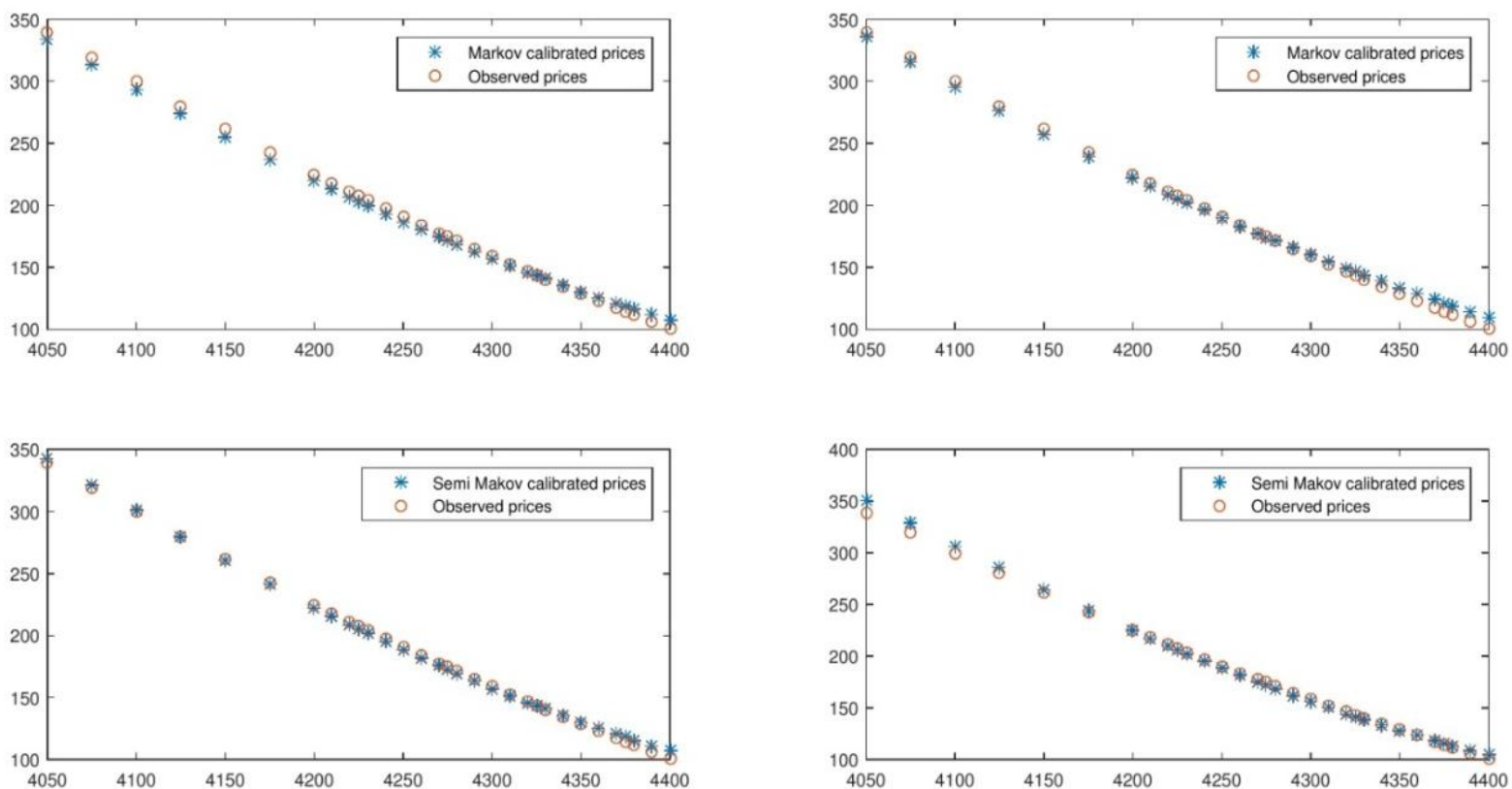

Figure 20. Calibration Results of the Markov and Semi Markov Regime Switching Models to NDX Observed Prices in 2015 Post Financial Crisis.

\subsection{Comparison with Heston Model}

In this section, we veer away from sequential calibration of option prices for each market maturity as performed in last section. We rather perform a full implied volatility surface calibration which we compare with the implied volatility surface generated by Markov regime switching Black Scholes and Heston models. The parameters of interest in Heston model as in most literature are $V_{0}$ the initial spot price volatility, $\kappa$ the speed of mean reversion of the spot price volatility, $\theta$ the long-term spot price volatility and $\rho$ the correlation of the Brownian motions driving the spot price and the Brownian motion driving the mean reverting volatility of the spot price. We recall that Heston volatility and Black Scholes models are two amongst the most used market models by practitioners in the financial market. Two of the main attractions of both models is their relative tractability as far as option pricing and the clarity of the economic interpretation of their calibrated parameters as well as their calibration performances relative to more complex models 
(Levy models for instance). We consider a full implied volatility surface of the semi Markov Black Scholes model, the Markov Black Scholes model and Heston model for a data set of option prices on the NASDAQ index quoted under the handle NDX and obtained from the website optioneducation.org. A summary of the calibration process is given on Table 6. It appears from rows 1, 2 and 4 of Table 6 that the semi Markov Black Scholes regime switching model fits a volatility surface as well and even slightly better than the Heston model (RMSE are 3.8 vs 4.6) and even better than Markov regime Black Scholes models (RMSE 3.8 vs 7.1). A closer look at the reason why the semi Markov Black Scholes outperforms Heston volatility surface is because of its ability to reproduce more accurately short term option prices as evidenced by Figure 21. Given that the basic Black Scholes model only supports flat volatility surfaces, one can attribute the added flexibility of the volatility surface generated by the semi Markov Black Scholes model to its switching nature which is in turn modeled as a semi Markov process. One can note the negligible contribution of the extra jump component at regime switches as the optimum parameters of the calibration algorithm leaves all four jump parameters equal to 0 and hence with no effect of option prices. Despite this encouraging model fit diagnostic of the semi Markov regime switching model, one cannot be oblivious to the lack of efficiency of its calibration algorithm compared to Heston and the Markov regime Black Scholes. Much research has been devoted to successfully improving on the efficiency of the Heston calibration model leading to efficient algorithm while the relative novelty of Semi Markov regime switching models in the derivative market is relatively unexplored, hence offering a decent research avenue. All algorithms used in this sections were implemented in MATLAB version R2016a and the global optimization tool was extensively used to avoid the additional bias of initial guesses in the comparison. The genetic and the simulated annealing algorithms were used to obtain an initial guess and find an optimum solution respectively.

Table 5. This table reports calibration results of model parameters for the Markov regimes Black Scholes (M), the semi Markov regimes Black Scholes (SM) and Heston models (HES) using option contracts on the NASDAQ (NDX) across the whole implied volatility surface (IVS) and short maturity options. We assume that the market has two regimes namely, $E=\{1,2\}$ and at inception of the contract, the market has been in its current state for $y=1.2 \mathrm{yrs}$.

\begin{tabular}{|c|c|c|c|c|c|c|c|c|c|c|c|c|c|c|}
\hline M & IVS & $\begin{aligned} & \hat{\alpha}_{1} \\
= & .313\end{aligned}$ & $\begin{array}{c}\hat{\alpha}_{2}= \\
14.30\end{array}$ & $\hat{\vartheta}_{1}=1$ & $\hat{\vartheta}_{2}=1$ & $\hat{\varepsilon}_{1}=0$ & $\hat{\varepsilon}_{2}=0$ & $\begin{array}{c}\hat{p}_{1}= \\
0\end{array}$ & $\begin{array}{c}\hat{p}_{2}= \\
0\end{array}$ & $\begin{aligned} & \sigma_{1} \\
= & .107\end{aligned}$ & $\begin{aligned} & \sigma_{2} \\
= & .122\end{aligned}$ & $\begin{array}{l}S S= \\
4933\end{array}$ & $\begin{array}{c}\mathrm{rmse}= \\
7.1\end{array}$ & $\begin{array}{c}\mathrm{CPU} \\
\text { time }=5 \mathrm{~h} \\
\text { ours }\end{array}$ \\
\hline SM & IVS & $\begin{array}{c}\hat{\alpha}_{1}= \\
39.716\end{array}$ & $\begin{array}{l}\hat{\alpha}_{2}= \\
8.699\end{array}$ & $\begin{array}{l}\hat{\vartheta}_{1}= \\
4.7\end{array}$ & $\begin{array}{c}\hat{\vartheta}_{2}= \\
8.058\end{array}$ & $\hat{\varepsilon}_{1}=0$ & $\hat{\varepsilon}_{2}=0$ & $\hat{p}_{1}=0$ & $\begin{array}{c}\hat{p}_{2}= \\
0\end{array}$ & $\begin{array}{c}\sigma_{1} \\
=.09\end{array}$ & $\begin{aligned} & \sigma_{2} \\
= & .172\end{aligned}$ & $\begin{array}{l}S S= \\
712\end{array}$ & $\begin{array}{c}\mathrm{rmse}= \\
3.8\end{array}$ & $\begin{array}{c}\mathrm{CPU} \\
\text { time }=22 \\
\text { hours }\end{array}$ \\
\hline & $\begin{array}{l}\text { Short } \\
\text { Time }\end{array}$ & $\begin{array}{c}\hat{\alpha}_{1}= \\
3.789\end{array}$ & $\begin{array}{c}\hat{\alpha}_{2}= \\
23.90\end{array}$ & $\begin{array}{c}\hat{\vartheta}_{1}= \\
54.82\end{array}$ & $\begin{array}{c}\hat{\vartheta}_{2.3} \\
53\end{array}$ & $\begin{array}{c}\hat{\varepsilon}_{1} \\
=.001 \\
=\end{array}$ & $\begin{aligned} & \hat{\varepsilon}_{2} \\
= & .019\end{aligned}$ & $\begin{aligned} & \hat{p}_{1} \\
= & .153\end{aligned}$ & $\begin{aligned} & \hat{p}_{2} \\
= & .003\end{aligned}$ & $\begin{array}{r}\sigma_{1}= \\
7.215\end{array}$ & $\begin{aligned} & \sigma_{2} \\
= & .053\end{aligned}$ & $\begin{array}{l}\mathrm{SS}= \\
244\end{array}$ & $\begin{array}{c}\mathrm{rmse}= \\
2.1\end{array}$ & $\begin{array}{l}\mathrm{CPU} \\
\text { time }=4 \mathrm{~h} \\
\text { ours }\end{array}$ \\
\hline HES & IVS & $\begin{aligned} & V_{0} \\
= & .017\end{aligned}$ & $\kappa=.12$ & $\begin{aligned} & \theta \\
= & .126\end{aligned}$ & $\sigma=.5$ & $\begin{aligned} & \rho \\
= & .207\end{aligned}$ & & & & & & $\begin{array}{l}\mathrm{SS}= \\
1010\end{array}$ & $\begin{array}{c}\mathrm{rmse}= \\
4.54\end{array}$ & $\begin{array}{c}\mathrm{CPU} \\
\text { time }=2 \mathrm{~h} \\
\text { ours }\end{array}$ \\
\hline & $\begin{array}{l}\text { Short } \\
\text { Time }\end{array}$ & $\begin{aligned} & V_{0} \\
= & .009\end{aligned}$ & $\begin{array}{c}\kappa= \\
2.438\end{array}$ & $\begin{aligned} & \theta \\
= & .139\end{aligned}$ & $\sigma .245$ & $\rho=.88$ & & & & & & $\begin{array}{l}\mathrm{SS}= \\
6104\end{array}$ & $\begin{array}{c}\text { rmse }= \\
10.7\end{array}$ & $\begin{array}{c}\mathrm{CPU} \\
\text { time }=.5 \mathrm{~h} \\
\text { ours }\end{array}$ \\
\hline
\end{tabular}



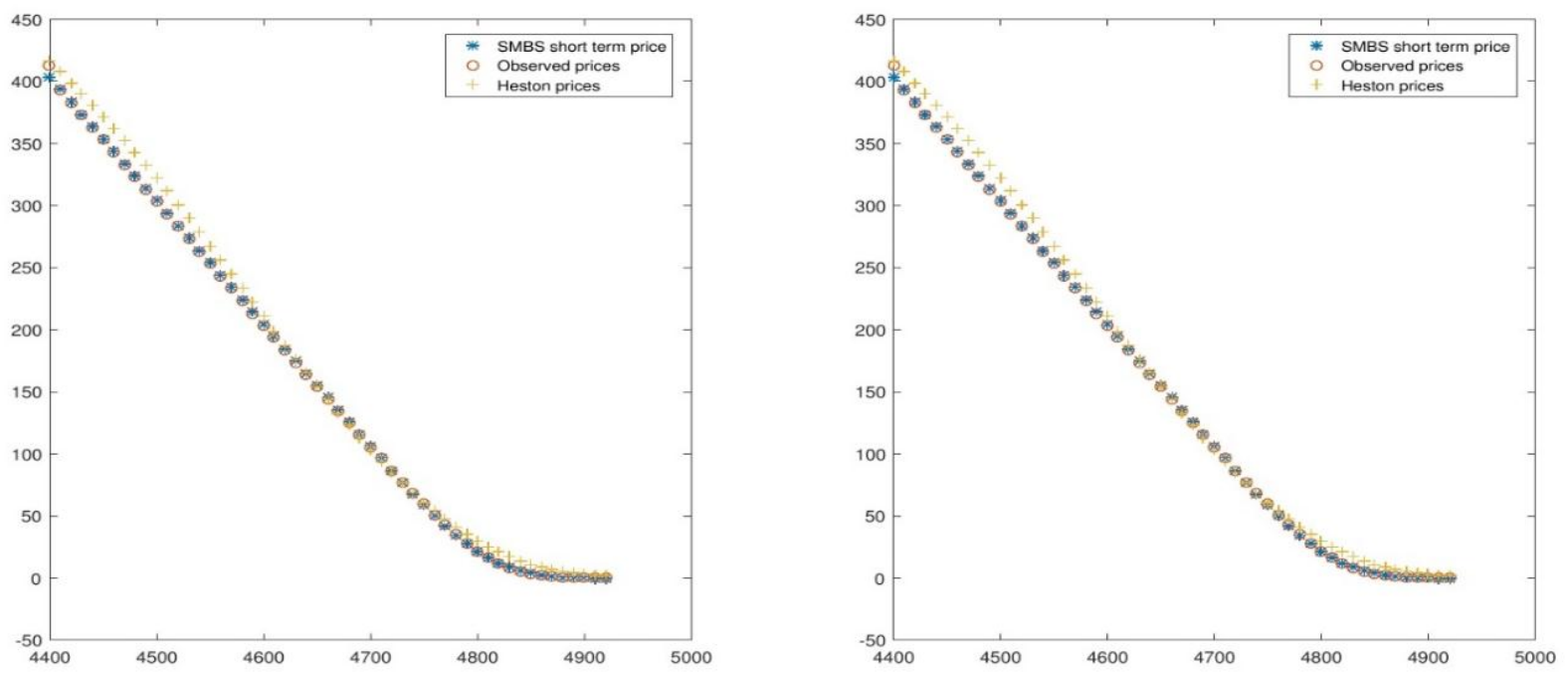

Figure 21. Comparison of Short Term NDX Prices Observed (August 2016), Generated from SMBS and Heston models. Both Figures Represent Option Prices Vs Strike prices

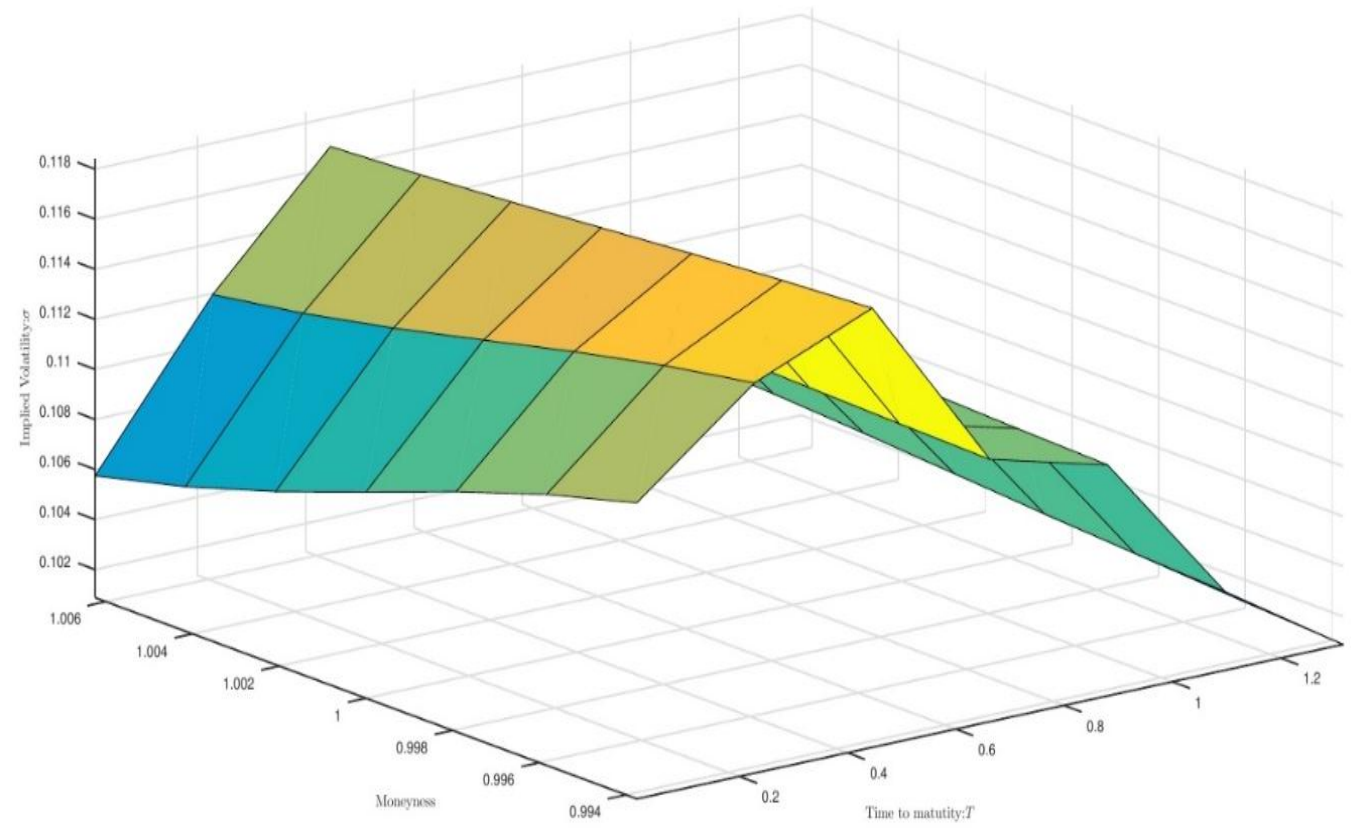

Figure 22. Implied Volatility Surface of NDX Observed Prices with the semi Markov Regime Switching Black Scholes Model in August 2016 as the Market Still Recovers from the 2008 Financial Crisis Assuming the Market in Regime 2. 


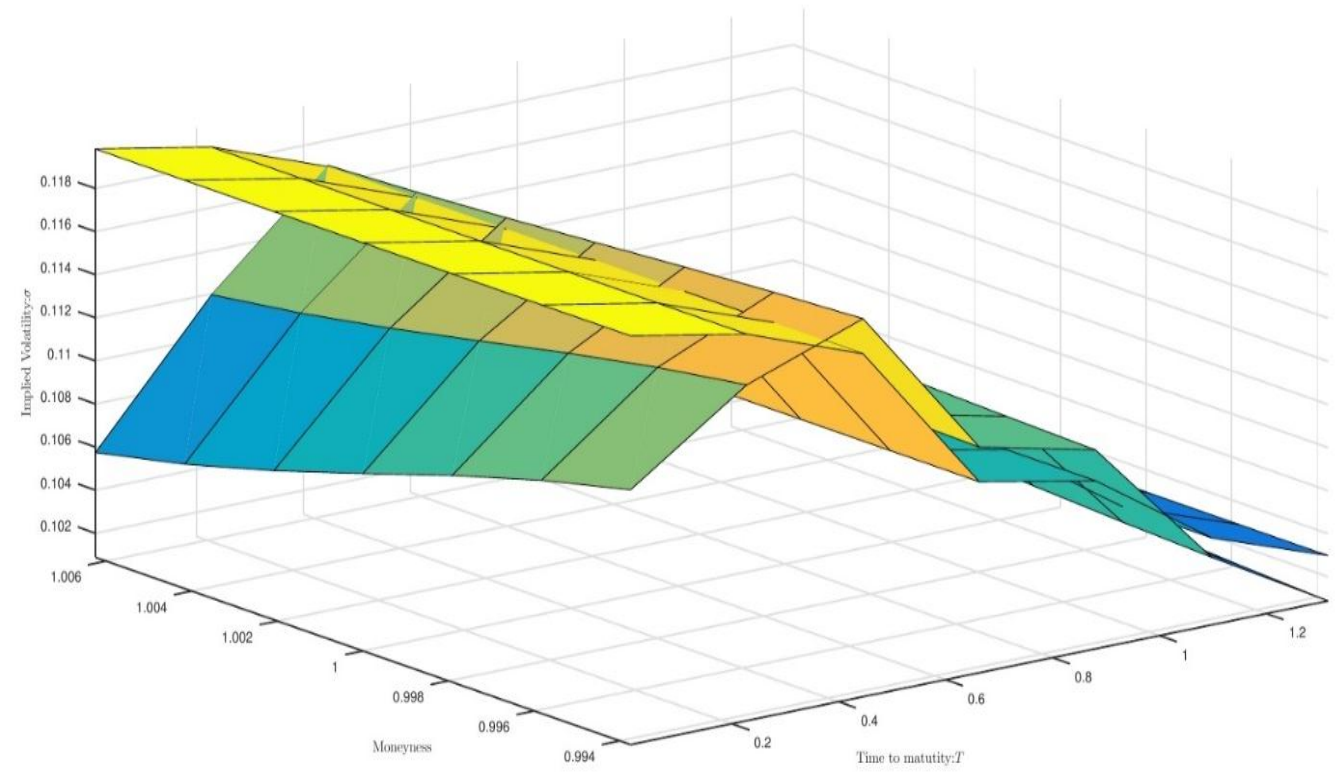

Figure 23. Implied Volatility Surface of NDX observed Prices with the Semi Markov Regime Switching Black Scholes Model in August 2016 as the Market Still Recovers from the 2008 financial Crisis Assuming the market in regime 1 along with Market Volatility Prices. The market Implied Volatility Surface is Blue-Green for short Maturities and the SMBS implied Volatility Surface is Yellow for Short Maturities.

\section{Conclusion}

In this paper, we have extended the ubiquitous Markov market regime model to a semi Markov market regime model in the context of option pricing. It allows a more accurate description of the risk neutral market regime dynamics as it assumes a time dependent conditional intensity of state changes. The main drawback is the increased complexity of the partial differential equation satisfied by the option price which translated in longer CPU times of calibration algorithms. We considered semi Markov processes with piecewise constant conditional intensity matrices, which allowed us to use Carr and Madan and the Fourier time stepping methods for option simulations and calibrations. An analysis of the semi Markov parameters effects on option prices shows that semi Markov parameters influence option prices to a visible extend, hence legitimizing the use of semi Markov regimes in derivative pricing. We performed a fit comparison of models with semi Markov and Markov markets regimes and showed that Black Scholes model under semi Markov regimes shows a slight improvement in sequential calibration (for each maturity) over Markov regime switching models and a substantial improvement in the calibration of full implied volatility surface over both Markov regime switching and Heston models. As previously mentioned, the runtime of the calibration algorithm is slower than Heston and Markov regime models, and every regime switching model (Markov or semi Markov) induces an incomplete market. Incompleteness of the market renders the risk neutral pricing argument more complex as there exist more than one risk neutral measures. This brings up the issue of choice of the risk neutral measure. In this article we used the minimal entropy martingale measure which is heuristically the risk neutral measure closest to the historical measure probability measure in the sense of Kulback Leibler distance, which minimizes the distance between the risk neutral and the historical view of the market. Another useful risk neutral measure is the minimal martingale measure which allows the best (with respect to certain risk functions) replication of option contracts for portfolio risk hedging purpose. Future research may consider the development of a minimal martingale measure for semi Markov regime switching models along with improving optimization of the calibration algorithm in semi Markov regimes.

\section{Acknowledgments}

This research was supported by the Mathematical Sciences Division, US Army Research Office by Grant No. W911NF-15-1-0182.

\section{References}

Anil, G. L., \& Ladde, G. S. (2013). An introduction to differential equations: Stochastic Modeling, Methods of analysis, 2. World Scientific Publishing Company, Singapore.

Bernt, K. Ø., \& Agnès, S. (2005). Applied stochastic control of jump diffusions, 498. 
Christian, R., \& George, C. (2013). Monte Carlo statistical methods. Springer Science \& Business Media.

Damien, D. (2007). On lévy processes for option pricing: Numerical methods and calibration to index options.

Daniel, S., \& Ladde, G. S. (2011). Stochastic hybrid system with non-homogeneous and boundary jumps. Nonlinear Analysis: Hybrid Systems, 5(3), 591-602. http://dx.doi.org/10.1016/j.nahs.2010.12.007

Donatien, H. (2010). Switching Lévy processes: a toolbox for financial applications. Technical report, CREST working paper.

Donatien, H., \& David, B. C. (2014). A structural model for credit risk with switching processes and synchronous jumps. The European Journal of Finance, 1-23. http://dx.doi.org/10.1080/1351847X.2014.924079.

Edwin, J. E., Martin, J. G., Stephen, J. B., \& William, N. G. (2009). Modern portfolio theory and investment analysis. John Wiley \& Sons.

Fischer, B., \& Myron, S. (1973). The pricing of options and corporate liabilities. The journal of political economy, 637654.

Grabbe, J. O. (1983). The pricing of call and put options on foreign exchange. Journal of International Money and Finance, 2(3), 239-253. http://dx.doi.org/10.1016/S0261-5606(83)80002-3.

Ingo, B. (2006). Application of hidden Markov models and Hidden Semi Markov models to financial time series. $\mathrm{PhD}$ thesis, Georg-August-University of Gottingen.

James, D. H. (1989). A new approach to the economic analysis of nonstationary time series and the business cycle. Econometrica: Journal of the Econometric Society, 357-384. http://www.jstor.org/stable/1912559.

Julien, H., \& Pierre, D. (2011b). Semi-markov regime switching interest rate models and minimal entropy measure. Physica A: Statistical Mechanics and its Applications, 390(21), 3767-3781. http://dx.doi.org/10.1016/j.physa.2011.04.036.

Kenneth, R. J., Sebastian, J., \& Vladimir, S. (2007). Option pricing with regime switching lévy processes using fourier space time stepping. In Proc. 4th IASTED Intern. Conf. Financial Engin. Applic, 92-97. http://dx.doi.org/10.2139/ssrn.1020209.

Kyriakos, C. (2002). Continuous time regime switching models and applications in estimating processes with stochastic volatility and jumps. U of London Queen Mary Economics Working Paper, 464.

Kyriakos, C. (2004). Option pricing using the fractional fft. Journal of Computational Finance, 8(2), 1-18. http://dx.doi.org/10.21314/JCF.2005.137.

Kyriakos, C.(2005). Switching lévy models in continuous time: Finite distributions and option pricing. University of Essex, Centre for Computational Finance and Economic Agents (CCFEA) Working Paper.

Massimo, C., Arturo, L., Ivar, M., \& Emilio, R. (2014). Option pricing under regime-switching jump-diffusion models. Journal of Computational and Applied Mathematics, 256, 152-167. http://dx.doi.org/10.1016/j.cam.2013.07.046.

Mrinal, K. G., \& Anindya, G.(2009). Risk minimizing option pricing in a semi-markov modulated market. SIAM Journal on control and Optimization, 48(3), 1519-1541. http://dx.doi.org/10.1137/080716839.

Nicolas, P. B. (1998). Valuing options in regime-switching models. The Journal of Derivatives, 6(1), 38-49. http://dx.doi.org/10.3905/jod.1998.408011.

Olusegun, M. O. (2014). Stochastic modeling and analysis of energy commodity spot price processes.

Olusegun, M. O. (2017). Local lagged adapted generalized method of moments and applications. Stochastic analysis and applications, 35 .

Patrick, A, \& Ladde, G.S. (2015). Option pricing with a levy-type stochastic dynamic model for stock price process under semi-markovian structural perturbations. International Journal of Theoretical and Applied Finance. http://dx.doi.org/10.1142/S0219024915500521.

Peter, C., \& Dilip, M. (1999). Option valuation using the fast fourier transform. Journal of computational finance, 2(4), 61-73. http://dx.doi.org/10.21314/JCF.1999.043.

Peter, T.(2003). Financial modelling with jump processes. CRC press.

Peter, E. K., \& Eckhard, P. (1992). Higher-order implicit strong numerical schemes for stochastic differential equations. Journal of statistical physics, 66(1-2), 283-314. http://dx.doi.org/10.1007/BF01060070.

Robert, J. E., \& Carlton-James, U. O. (2006). Option pricing for pure jump processes with markov switching compensators. Finance and Stochastics, 10(2), 250-275. http://dx.doi.org/10.1007/s00780-006-0004-6. 
Robert, J. E., Leunglung, C., \& Tak, K. S.(2005). Option pricing and esscher transform under regime switching. Annals of Finance, 1(4), 423-432. http://dx.doi.org/423. doi:10.1007/s10436-005-0013-z.

Romuald, M. (2012). Les processus additifs Markoviens et leurs applications en finances Mathematiques. PhD thesis, Universite de Montreal.

Sovan, M. (2009). Regime switching stochastic volatility with perturbation based option pricing. arXiv preprint arXiv:0904.1756.

Vasanttilak, N. (1993). Option valuation and hedging strategies with jumps in the volatility of asset returns. The Journal of Finance, 48(5), 1969-1984. http://dx.doi.org/10.1111/j.1540-6261.1993.tb05137.x.

Vladimir, S. R. J., \& Sebastien, J. (2009). Fourier space time-stepping for option pricing with levy models. The journal of computational finance, 12 .

Wilhelmm, M.(1954). On the exponential solution of differential equations for a linear operator. Communications on pure and applied mathematics, 7(4), 649-673. http://dx.doi.org/10.1002/cpa.3160070404.

Wu, L., \& Ladde, G.S (2009a). Stochastic modeling and statistical analysis on the stock price process, Nonlinear analysis theory and methods, 71, 1203-1208.

Wu, L., \& Ladde, G. S (2009b). Development of Nonlinear stochastic models by using stock price data and basic statistics, Neural parallel and scientific computation, 18, 269-282

$\mathrm{Wu}$, L. (2010). Stochastic modeling and statistical analysis. PhD thesis, University of South Florida.

\section{Copyrights}

Copyright for this article is retained by the author(s), with first publication rights granted to the journal.

This is an open-access article distributed under the terms and conditions of the Creative Commons Attribution license which permits unrestricted use, distribution, and reproduction in any medium, provided the original work is properly cited. 